\title{
On First Occurrence in the Local Theta Correspondence
}

\author{
Stephen S. Kudla* \\ and \\ Stephen Rallis ${ }^{\dagger}$
}

\begin{abstract}
This paper discusses a conservation conjecture for the first occurrence indices. Such indices record the first occurrence of an irreducible admissible representation $\pi$ of a fixed group $G$ in the local theta correspondence as the second member of a dual pair $\left(G, G^{\prime}\right)$ runs over the isometry groups in a Witt tower. For example, for a fixed nonarchimedean local field $F$ and for a fixed quadratic character $\chi$ of $F^{\times}$, there are two Witt towers $\left\{V_{m}^{+}\right\}$and $\left\{V_{m}^{-}\right\}$, where the quadratic space $V_{m}^{ \pm}$has dimension $m$, character $\chi$ and Hasse invariant \pm 1 . If $m_{\chi}^{ \pm}(\pi)$ is the dimension of the space $V_{m}^{ \pm}$for which the irreducible admissible representation $\pi$ of $\operatorname{Sp}_{n}(F)$ first occurs in the theta correspondence for the dual pair $\left(S p_{n}(F), O\left(V_{m}^{ \pm}\right)\right)$, then we conjecture that

$$
m_{\chi}^{+}(\pi)+m_{\chi}^{-}(\pi)=4 n+4 .
$$
\end{abstract}

We prove this conjecture in many cases, in particular, when $\pi$ is a supercuspidal representation.

2000 Mathematics Subject Classification: primary 11F27; secondary 22E50.

\section{Introduction}

A basic step in understanding the local theta correspondence is the description of the behavior of the correspondence as one group in the reductive dual pair varies in a Witt tower, [18]. The combination of this Witt tower technique, with the method of 'doubling', [4], [18], and the local 'doubling' zeta integrals, [16], [17], lies behind much of our qualitative understanding of the correspondence. In this paper $^{1}$, in the case of a nonarchimedean local field,

\footnotetext{
*Partially supported by NSF Grant DMS-0200292 and by a Max-Planck Research Prize from the Max-Planck Society and Alexander von Humboldt Stiftung.

$\dagger$ Partially supported by NSF Grant DMS 0140422.

${ }^{1}$ which has languished in a drawer for nearly 10 years, due to the idolence of the first author
} 
we conjecture a 'conservation relation' between the first occurrence indices in a pair of related Witt towers and prove it in many cases.

Suppose that $F$ is a nonarchimedean local field of characteristic zero. Two quadratic spaces $V$ and $V^{\prime}$ over $F$ are said to lie in the same Witt tower if they are isomorphic after the addition of a suitable number of hyperbolic planes. The Witt tower of $V$ is then determined by the discriminant character $\chi=\chi_{V}$ and the Hasse invariant $\epsilon=\epsilon(V)= \pm 1$, and, of course, the parity of the dimension of $V$. If $\chi$, a quadratic character of $F^{\times}$, and a parity have been fixed, there are two associated Witt towers $\left\{V_{m}^{+}\right\}$and $\left\{V_{m}^{-}\right\}$, where $\chi_{V_{m}^{ \pm}}=\chi$, $\epsilon\left(V_{m}^{ \pm}\right)= \pm 1$, and $\operatorname{dim}_{F}\left(V_{m}^{ \pm}\right)=m$. Let $G_{n}^{\prime}=\operatorname{Sp}\left(W_{n}\right)$ be the symplectic group of rank $n$ over $F$, in the case of even parity, or $\operatorname{Mp}\left(W_{n}\right)$, its metaplectic extension, in the case of odd parity. Let $\operatorname{Irr}\left(G_{n}^{\prime}\right)$ be the set of isomorphism classes of irreducible admissible (genuine) representations of $G_{n}^{\prime}$. For $\pi \in$ $\operatorname{Irr}\left(G_{n}^{\prime}\right)$, the first occurrence index $m_{\chi}^{ \pm}(\pi)$ is the smallest dimension $m$ for which $\pi$ occurs in the local theta correspondence for the dual pair $\left(G_{n}^{\prime}, O\left(V_{m}^{ \pm}\right)\right)$. It is known that $\pi$ then occurs in the correspondence for any pair $\left(G_{n}^{\prime}, O\left(V_{m}^{ \pm}\right)\right)$with $m \geq m_{\chi}^{ \pm}(\pi)$, and that, by Howe's stable range condition, $m_{\chi}^{ \pm}(\pi) \leq m_{0}+2 n$, where $m_{0}=m_{0}( \pm, \chi)$ is the dimension of the smallest quadratic space in the tower $\left\{V_{m}^{ \pm}\right\}$. The conservation relation can be stated as follows.

Conjecture A. For any $\pi \in \operatorname{Irr}\left(G_{n}^{\prime}\right)$,

$$
m_{\chi}^{+}(\pi)+m_{\chi}^{-}(\pi)=4 n+4 .
$$

In the case of odd parity, Conjecture A implies the following theta dichotomy phenomenon:

Conjecture B. Fix a quadratic character $\chi$. Any $\pi \in \operatorname{Irr}\left(G_{n}^{\prime}\right)$ occurs in the theta correspondence with precisely one of the spaces $V_{2 n+1}^{+}$and $V_{2 n+1}^{-}$.

In fact, this dichotomy is controlled by a local root number, cf. [3] in the unitary group case, and this phenomenon leads to interesting global arithmetic related to central values and derivatives of L-functions, [8]. We will not discuss these aspects further in the present paper, see, however, [11].

Conjecture A asserts that if a representation $\pi$ occurs 'early' in one tower, it must occur late in the other. The most extreme example of this is the case of the trivial representation $\mathbb{1}$ of $\operatorname{Sp}\left(W_{n}\right)$, which we view (formally) as the Weil representation of the zero dimensional space at the bottom of Witt tower of split quadratic space $\left\{V_{m}^{+}\right\}$for the trivial character $\chi=\chi_{0}=1$. Thus, $m_{\chi_{0}}^{+}(\mathbb{1})=0$. On the other hand, we show:

Theorem 1. The conservation relation holds for $\pi=\mathbb{1 1}$ and for any quadratic character $\chi$. In fact, $m_{\chi_{0}}^{-}(\mathbb{1})=4 n+4$, and $m_{\chi}^{ \pm}(\mathbb{1})=2 n+2$, for $\chi \neq \chi_{0}$. 
This result is actually an essential intermediate step in obtaining the following criterion in the general case. Let

$$
\iota: G_{n}^{\prime} \times G_{n}^{\prime} \rightarrow G_{2 n}^{\prime}
$$

be the doubling embedding, and let $I_{2 n}^{\prime}(s, \chi)$ be the degenerate principal series representation of $G_{2 n}^{\prime}$ defined by the character $\chi$ and $s \in \mathbb{C}$.

Theorem 2. Fix a quadratic character $\chi$ and a parity. Suppose that $\pi$ is an irreducible admissible representation of $G_{n}^{\prime}$ such that

$$
\operatorname{dim} \operatorname{Hom}_{G_{n}^{\prime} \times G_{n}^{\prime}}\left(I_{2 n}\left(s_{0}, \chi\right), \pi \otimes \pi^{\vee}\right)=1,
$$

for all $s_{0}$ in the set:

$$
\text { Crit }= \begin{cases}\left\{-n-\frac{1}{2},-n+\frac{1}{2}, \ldots, n-\frac{1}{2}, n+\frac{1}{2}\right\} & \text { for even parity and } \chi=1, \\ \left\{-n+\frac{1}{2}, \ldots, n-\frac{1}{2}\right\} & \text { for even parity and } \chi \neq 1, \\ \{-n,-n+1, \ldots, n-1, n\} & \text { for odd parity. }\end{cases}
$$

Then

$$
m_{\chi}^{+}(\pi)+m_{\chi}^{-}(\pi)=4 n+4 .
$$

Of course, many representations $\pi \in \operatorname{Irr}\left(G_{n}^{\prime}\right)$ satisfy the condition of this Theorem. For example:

Corollary 3. The conservation relation of Conjecture $A$ holds for any supercuspidal representation $\pi \in \operatorname{Irr}\left(G_{n}^{\prime}\right)$. In particular, in the metaplectic case, such representations satisfy theta dichotomy.

Analogous conservations relations should hold for other dual pairs.

For example, for an irreducible admissible representation $\tau \in \operatorname{Irr}(O(V))$ of the orthogonal group of some quadratic space $V$ over $F$, let $n(\tau)$ be the smallest $n$ for which $\tau$ occurs in the theta correspondence for the dual pair $\left(O(V), G_{n}^{\prime}\right)$. Again, we know that $\tau$ then occurs in the correspondence for the pair $\left(O(V), G_{n}^{\prime}\right)$ for any $n \geq n(\tau)$, and that $n(\tau) \leq m=\operatorname{dim}_{F}(V)$. Let $\nu: O(V) \rightarrow F^{\times} / F^{\times, 2}$ be an extension to $O(V)$ of the spinor norm on $S O(V)$. For any quadratic character $\chi$ of $F^{\times}$, let $\tilde{\chi}=\chi \circ \nu$ be the corresponding character of $O(V)$ and let $\operatorname{sgn} \cdot \tilde{\chi}$ be its twist by the $\operatorname{sgn}$ character. The analogue of the conservation relation in this case is the following.

Conjecture C. For a fixed quadratic character $\chi$ and for any $\pi \in \operatorname{Irr}(O(V))$,

$$
n(\tilde{\chi} \cdot \tau)+n(\operatorname{sgn} \cdot \tilde{\chi} \cdot \tau)=\operatorname{dim}_{F}(V) .
$$

The analogous conjectures in the case of unitary groups are made in [3]. The methods of the present paper can be applied in the orthogonal and unitary cases as well. We hope to discuss the results elsewhere. 
Since this paper was first written, a number of people have established conservation relations and dichotomy in various cases. For example, for unitary groups over $\mathbb{R}$, quite precise information about the correspondence was obtained by A. Paul, [15] and the conservation relations were verified ${ }^{2}$. In the nonarchimedean case, there is work of Muiç, [14], who proves the conservation relation for discrete series representations $\pi \in \operatorname{Irr}_{d s}\left(G_{n}^{\prime}\right)$ in the case of even parity, using the work of Moeglin and Tadic [12].

The authors were aware of elementary cases of dichotomy, a number of which are contained in [18]. This lead to some of the problems studied below. In particular, the conservation conjecture stated above and its analogue for unitary groups were found by the first author while visiting the Tata Institute in Bombay in January 1994. A serious effort to try to prove these conjectures was undertaken jointly soon thereafter, and the present paper, which utilizes in large part the methods of [18], was essentially written by May of 1995 . The results were summarized in the lecture notes [7] in 1996. The present volume seemed like a good occasion to finally finish the task of polishing it for publication. The first author would like to express his heartfelt appreciation to the second for all of the mathematics and encouragment he has shared so generously over the years.

\section{Quotients of degenerate principal series}

In this section we recall some of the basic facts about certain degenerate principal series representations and their restrictions via doubling.

Let $F$ be a non-archimedean local field of characteristic 0 , and let $W,<,>$ be a non-degenerate symplectic vector space of dimension $2 n$ over $F$. We fix an identification $W=F^{2 n}$ (row vectors) such that

$$
<w_{1}, w_{2}>=w_{1} J^{t} w_{2}, \quad J=\left(\begin{array}{cc} 
& 1_{n} \\
-1_{n} &
\end{array}\right),
$$

and we view the symplectic group $G=G_{n}=S p(W)$ as acting on $W$ on the right. We let $e_{1}, \ldots, e_{n}, e_{1}^{\prime}, \ldots, e_{n}^{\prime}$ be the fixed basis of $W$. Let $P_{n}$ be the stabilizer of the maximal isotropic subspace of $W$ spanned by the rows of the matrix $\left(0,1_{n}\right) \in M_{n, 2 n}(F)$, i.e., by the vectors $e_{1}^{\prime}, \ldots, e_{n}^{\prime}$, and let

$$
M=\left\{m(a)=\left(\begin{array}{ll}
a & \\
& { }^{t} a^{-1}
\end{array}\right) \mid a \in \mathrm{GL}_{n}(F)\right\},
$$

and

$$
N=\left\{n(b)=\left(\begin{array}{ll}
1 & b \\
& 1
\end{array}\right) \mid b={ }^{t} b \in \mathrm{M}_{n}(F)\right\},
$$

\footnotetext{
${ }^{2}$ Here there are many Witt towers and one must group them suitably.
} 
so that $P_{n}=M N$ is a Levi decomposition of the Siegel parbolic $P_{n}$. Let $K=S p_{n}(\mathcal{O})$ be the maximal compact subgroup stabilizing the lattice $\mathcal{O}^{2 n}$ in $W$. In the Iwasawa decomposition $G=P K=N M K$, write $g=n m(a) k$, and note that the quantity

$$
|a(g)|=|\operatorname{det} a|
$$

is well defined, independent of the choice of $a \in \mathrm{GL}_{n}(F)$. Similarly, for any $r, 1 \leq r \leq n$, let $P_{r}$ be the maximal parabolic of $G_{n}$ which stabilizes the isotropic $r$-plane spanned by the first $r$ rows of the matrix $\left(0,1_{n}\right)$, i.e., by the vectors $e_{1}^{\prime}, \ldots, e_{r}^{\prime}$. Then there is a Levi decomposition $P_{r}=M_{r} N_{r}$ where $M_{r} \simeq \mathrm{GL}_{r} \times G_{n-r}$ is the subgroup of $P_{r}$ which stabilizes the span of the first $r$ rows of the matrix $\left(1_{n}, 0\right)$ and where

$$
N_{r}=\left\{\left(\begin{array}{cccc}
1_{r} & x & z & y \\
& 1_{n-r} & { }^{t} y & 0 \\
& & 1_{r} & \\
& & -{ }^{t} x & 1_{n-r}
\end{array}\right)\right\} .
$$

Now let

$$
\mathbb{W}=W+W_{-},
$$

where $W_{-}=W$ with symplectic form $-<$, >. Let $H=S p(\mathbb{W})$, and note that, for a suitable choice of basis, we have $H \simeq G_{2 n}$, the natural map

$$
\iota: G_{n} \times G_{n} \longrightarrow H=G_{2 n}
$$

is given by

$$
\iota\left(g_{1}, g_{2}\right)=\iota_{0}\left(g_{1}, g_{2}^{\vee}\right)
$$

with

$$
\iota_{0}\left(\left(\begin{array}{ll}
a_{1} & b_{1} \\
c_{1} & d_{1}
\end{array}\right),\left(\begin{array}{ll}
a_{2} & b_{2} \\
c_{2} & d_{2}
\end{array}\right)\right)=\left(\begin{array}{llll}
a_{1} & & b_{1} & \\
& a_{2} & & b_{2} \\
c_{1} & & d_{1} & \\
& c_{2} & & d_{2}
\end{array}\right)
$$

and

$$
g^{\vee}=\left(\begin{array}{ll}
1 & \\
& -1
\end{array}\right) g\left(\begin{array}{ll}
1 & \\
& -1
\end{array}\right)^{-1}
$$

Let $P=P_{2 n} \subset H$ be the Siegel parabolic in $H$. For $s \in \mathbb{C}$ and for a (unitary) character $\chi$ of $F^{\times}$, let

$$
I_{2 n}(s, \chi)=I_{P}^{H}\left(\chi||^{s}\right)
$$


be the degenerate principal series representation of $H$ consisting of smooth complex valued functions $\Phi(s)$ on $H$ (sections) satisfying

$$
\Phi(n m(a) h, s)=\chi(a)|a|^{s+\rho_{2 n}} \Phi(h, s),
$$

where we write $\chi(a)$ for $\chi(\operatorname{det}(a))$ and where $\rho_{2 n}=n+\frac{1}{2}$. Such a section is called standard for its restriction to $K$ is independent of $s$. Information about the points of reducibility of these representations and their composition series can be found in [2], [9], [20].

The proof of the following uniqueness result plays a fundamental role. Its proof will be give in the next section.

Theorem 1.1. Let $\mathbb{1 1}$ be the trivial representation of $G \times G$. For all $s \in \mathbb{C}$ and for all characters $\chi$,

$$
\operatorname{dim} \operatorname{Hom}_{G \times G}\left(I_{2 n}(s, \chi), \mathbb{1}\right)=1 .
$$

Here $I_{2 n}(s, \chi)$ is viewed as a representation of $G \times G$ via $\iota$.

In fact, we believe the following:

Conjecture 1.2. For all irreducible admissible representations $\pi$ of $G$, and for all $s$ and $\chi$,

$$
\operatorname{dim} \operatorname{Hom}_{G \times G}\left(I_{2 n}(s, \chi), \pi \otimes \pi^{\vee}\right)=1 .
$$

In the remainder of this section, we review some of the facts which are involved in the proofs.

The geometry of the double cosets for $P$ and $G \times G$ in $H$, or, equivalently, of the $G \times G$ orbits in the symplectic Grassmannian $P \backslash H$ of isotropic $2 n$-planes in $\mathbb{W}$ is well known [17], [7]. Recall that there is a double coset decomposition

$$
H=\coprod_{r=0}^{n} P \delta_{r} \iota(G \times G),
$$

where the image of

$$
\Omega_{r}:=P \delta_{r} \iota(G \times G)
$$

in $P \backslash H$ consists of those isotropic $2 n$-planes $U$ in $\mathbb{W}$ whose intersections $U \cap W$ and $U \cap W_{-}$have dimension $r$. Moreover, the closure of an orbit is given by

$$
\bar{\Omega}_{r}=\coprod_{j \geq r} \Omega_{j} .
$$

We write

$$
\mathrm{St}_{r}:=\iota^{-1}\left(\left(\delta_{r}^{-1} P \delta_{r}\right) \cap \iota(G \times G)\right)
$$


for the stabilizer of the point $P \cdot \delta_{r}$ in $P \backslash H$, and we choose the orbit representatives $\delta_{r}$ as follows. First, for the open orbit $\Omega_{0}$, let

$$
\delta_{0}=\delta_{0}^{(n)}=\left(\begin{array}{cccc}
0 & & 1 & \\
& 1 & & 0 \\
-1 & 1 & 0 & \\
& 0 & 1 & 1
\end{array}\right)
$$

where the blocks have size $n \times n$. For this choice of orbit representative,

$$
\mathrm{St}_{0}=\Delta(G),
$$

the diagonal in $G \times G$. For the closed orbit $\Omega_{n}=P \delta_{n} \iota(G \times G)$, we take $\delta_{n}=1$, so that

$$
\mathrm{St}_{n}=P_{n} \times P_{n} \subset G \times G,
$$

where $P_{n} \subset G_{n}=G$ is the Siegel parabolic of $G$. Finally, for any $r$, let $W=W^{\prime}+W^{\prime \prime}$ where $W^{\prime}$ is the span of the vectors $e_{1}, \ldots, e_{r}, e_{1}^{\prime}, \ldots, e_{r}^{\prime}$ and $W^{\prime \prime}$ is the span of the vectors $e_{r+1}, \ldots, e_{n}, e_{r+1}^{\prime}, \ldots, e_{n}^{\prime}$. Then consider the decomposition

$$
\mathbb{W}=\mathbb{W}^{\prime}+\mathbb{W}^{\prime \prime}
$$

with $\mathbb{W}^{\prime}=W^{\prime}+W_{-}^{\prime}$ and $\mathbb{W}^{\prime \prime}=W^{\prime \prime}+W_{-}^{\prime \prime}$. There is a corresponding imbedding

$$
S p\left(\mathbb{W}^{\prime}\right) \times S p\left(\mathbb{W}^{\prime \prime}\right) \longrightarrow H
$$

and we let $\delta_{r}$ be the image $1_{\mathbb{W}^{\prime}} \times \delta_{0, \mathbb{W}^{\prime \prime}}$. Explicitly, this is

$$
\delta_{r}=\left(\begin{array}{cccccccc}
1 & & & & 0 & & & \\
& 0 & & & & 1 & & \\
& & 1 & & & & 0 & \\
& & & 1 & & & & 0 \\
0 & & & & 1 & & & \\
& -1 & & 1 & & 0 & & \\
& & 0 & & & & 1 & \\
& & & 0 & & 1 & & 1
\end{array}\right)
$$

Note that this choice corresponds to an isotropic $2 n$-plane in $\mathbb{W}$ which intersects $W^{\prime}$ and $W_{-}^{\prime}$ in isotropic $r$-planes and which is transverse to $W^{\prime \prime}$ and $W_{-}^{\prime \prime}$. It is then not difficult to check that

$$
\mathrm{St}_{r}=\left(\mathrm{GL}_{r} \times \mathrm{GL}_{r} \times \Delta\left(G_{n-r}\right)\right) \ltimes\left(N_{r} \times N_{r}\right) \subset P_{r} \times P_{r} .
$$


Later it will be useful to know that, if $\gamma=\left(a_{1}, a_{2},\left(\begin{array}{ll}a & b \\ c & d\end{array}\right)\right) \in \operatorname{St}_{r}$, with $a_{1}$ and $a_{2} \in \mathrm{GL}_{r}$ and $\left(\begin{array}{ll}a & b \\ c & d\end{array}\right) \in G_{n-r}$, then

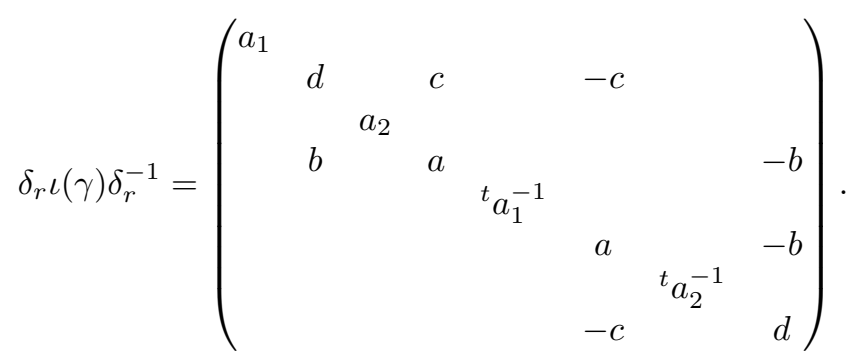

The stratification of $H$ by the orbits $\Omega_{r}$ gives rise to a filtration

$$
I_{2 n}(s, \chi)=I_{2 n}(s, \chi)^{(n)} \supset \cdots \supset I_{2 n}(s, \chi)^{(r)} \supset \cdots \supset I_{2 n}(s, \chi)^{(0)},
$$

where $I_{2 n}(s, \chi)^{(r)}$ is the set of functions in $I_{2 n}(s, \chi)$ whose restriction to the closure of $\Omega_{r+1}$ vanishes. The successive quotients are then induced representations of $G \times G$ given by

$$
\begin{aligned}
Q_{r}(s, \chi) & :=I_{2 n}(s, \chi)^{(r)} / I_{2 n}(s, \chi)^{(r+1)} \\
& \simeq I_{P_{r} \times P_{r}}^{G \times G}\left(\chi||^{s+\frac{r}{2}} \otimes \chi||^{s+\frac{r}{2}} \otimes S\left(G_{n-r}\right)\right),
\end{aligned}
$$

where the induction is normalized. Here we are inducing from the representation of $P_{r} \times P_{r}$ in which $N_{r} \times N_{r}$ acts trivially, the factor $\mathrm{GL}_{r} \times \mathrm{GL}_{r}$ acts by the character $\left.\left.\chi\right|^{s+\frac{r}{2}} \otimes \chi\right|^{s+\frac{r}{2}}$, and the factor $G_{n-r} \times G_{n-r}$ acts by left-right multiplication on the Schwartz space $S\left(G_{n-r}\right)$. In particular,

$$
Q_{0}(s, \chi) \simeq S(G),
$$

is a subrepresentation of $I_{2 n}(s, \chi)$, and

$$
Q_{n}(s, \chi) \simeq I_{n}\left(s+\frac{n}{2}, \chi\right) \otimes I_{n}\left(s+\frac{n}{2}, \chi\right)
$$

is a quotient of $I_{2 n}(s, \chi)$.

Definition 1.3. For $\chi$ fixed, an irreducible admissible representation $\pi$ of $G$ is said to occur on the boundary at the point $s$ if

$$
\operatorname{Hom}_{G \times G}\left(Q_{r}(s, \chi), \pi \otimes \pi^{\vee}\right) \neq 0,
$$

for some $r>0$.

Since the matrix coefficients of any irreducible admissible representation $\pi$ of $G$ lie in $C^{\infty}(G)=\operatorname{Ind} d_{1}^{G}(\mathbb{1})$, the contragradient of $S(G)=i n d_{1}^{G}(\mathbb{1})$, it 
follows that every representation occurs with multiplicity one on the open cell, i.e.,

Lemma 1.4. For any fixed $\chi$ and $s$, and for any irreducible admissible representation $\pi$ of $G$,

$$
\operatorname{dim} \operatorname{Hom}_{G \times G}\left(Q_{0}(s, \chi), \pi \otimes \pi^{\vee}\right)=\operatorname{dim} \operatorname{Hom}_{G \times G}\left(S(G), \pi \otimes \pi^{\vee}\right)=1 .
$$

When $r>0$, we apply Frobenius reciprocity to obtain:

$$
\begin{aligned}
& \operatorname{Hom}_{G \times G}\left(Q_{r}(s, \chi), \pi \otimes \pi^{\vee}\right) \\
& \quad \simeq \operatorname{Hom}_{G \times G}\left(\pi^{\vee} \otimes \pi, I_{P_{r} \times P_{r}}^{G \times G}\left(\chi^{-1}||^{-s-\frac{r}{2}} \otimes \chi^{-1}||^{-s-\frac{r}{2}} \otimes C^{\infty}\left(G_{n-r}\right)\right)\right) \\
& \quad \simeq \operatorname{Hom}_{M_{r} \times M_{r}}\left(\left(\pi^{\vee}\right)_{N_{r}} \otimes \pi_{N_{r}}, \chi^{-1}||^{-s-\frac{r}{2}} \otimes \chi^{-1}||^{-s-\frac{r}{2}} \otimes C^{\infty}\left(G_{n-r}\right)\right) .
\end{aligned}
$$

Here $M_{r} \simeq \mathrm{GL}_{r} \times \mathrm{GL}_{r} \times G_{n-r} \times G_{n-r}$. Just as in the case of the open orbit, if $\pi_{N_{r}}$ is non-zero, the restriction of $\left(\pi^{\vee}\right)_{N_{r}} \otimes \pi_{N_{r}}$ to $G_{n-r} \times G_{n-r}$ always has a non-zero intertwining map to $C^{\infty}\left(G_{n-r}\right)$. Thus, occurrence on the boundary is controlled by the spaces $\operatorname{Hom}_{\mathrm{GL}_{r}}\left(\left(\pi^{\vee}\right)_{N_{r}}, \chi^{-1}||^{-s-\frac{r}{2}}\right)$ and $\operatorname{Hom}_{\mathrm{GL}_{r}}\left(\pi_{N_{r}}, \chi^{-1}||^{-s-\frac{r}{2}}\right)$.

Lemma 1.5. An irreducible admissible representation $\pi$ of $G$ occurs on the boundary at the point $s$ if and only if, for some $r$, with $1 \leq r \leq n$,

$$
\operatorname{Hom}_{\mathrm{GL}_{r}}\left(\left(\pi^{\vee}\right)_{N_{r}}, \chi^{-1}||^{-s-\frac{r}{2}}\right) \neq 0
$$

and

$$
\operatorname{Hom}_{\mathrm{GL}_{r}}\left(\pi_{N_{r}}, \chi^{-1}||^{-s-\frac{r}{2}}\right) \neq 0
$$

Next, we recall the basic doubling integral [16] associated to an irreducible admissible representation $\pi$ of $G$. Let $\phi(g)=<\pi^{\vee}(g) \xi^{\vee}, \xi>$, for $\xi \in \pi$ and $\xi^{\vee} \in \pi^{\vee}$, be a matrix coefficient of $\pi^{\vee}$. Then, for any section $\Phi(s) \in I_{2 n}(s, \chi)$, let

$$
Z(s, \chi, \phi, \Phi):=\int_{G} \phi(g) \Phi\left(\delta_{0} \iota(g, 1), s\right) d g .
$$

In [16] it is proved that this integral converges for $\operatorname{Re}(s)$ sufficiently large, and that, for fixed data $\phi$ and $\Phi(s)$, with $\Phi(s)$ standard, $Z(s, \chi, \phi, \Phi)$ has a meromorphic continuation to the whole $s$ plane and is given by a rational function of $q^{-s}$. Moreover, the family of such functions obtained by varying $\phi$ and $\Phi(s)$ has a gcd of the form $f\left(q^{-s}\right)^{-1}$ for some polynomial $f(x)$. Since the representation of $G \times G$ on $I_{2 n}(s, \chi)$ is smooth, $Z\left(s_{0}, \chi, \cdot, \cdot\right)$ defines an element of

$$
Z\left(s_{0}, \chi\right) \in \operatorname{Hom}_{G \times G}\left(I_{2 n}\left(s_{0}, \chi\right), \pi \otimes \pi^{\vee}\right)
$$


at any value $s_{0}$ for which $f\left(q^{-s_{0}}\right) \neq 0$. In general, the leading term of the Laurent expansion of $Z(s, \chi, \cdot, \cdot)$ at $s=s_{0}$ defines an element

$$
Z^{*}\left(s_{0}, \chi\right) \in \operatorname{Hom}_{G \times G}\left(I_{2 n}\left(s_{0}, \chi\right), \pi \otimes \pi^{\vee}\right) .
$$

Next suppose that $\chi$ is unramified and note that there is, then, a unique standard section $\Phi^{0}(s)$ determined by the condition $\Phi(k, s)=1$ for all $k \in K_{H}$. Suppose that $\pi=\pi(\lambda)$ is the unramified representation of $G$ with Satake parameter

$\operatorname{diag}\left(q^{\lambda_{1}}, q^{\lambda_{2}}, \ldots, q^{\lambda_{n}}, 1, q^{-\lambda_{1}}, q^{-\lambda_{2}}, \ldots, q^{-\lambda_{n}}\right) \times F r \in \mathrm{SO}(2 n+1, \mathbb{C}) \times W_{F}={ }^{L} G$.

Let

$$
\phi^{0}(g)=<\pi^{\vee}(g) \xi_{0}^{\vee}, \xi_{0}>
$$

be the unramified matrix coefficient of $\pi^{\vee}$, with $\phi^{0}(e)=1$. Here $\xi_{0}\left(\right.$ resp. $\xi_{0}^{\vee}$ ) is a $K$-fixed vector in $\pi\left(\right.$ resp. $\left.\pi^{\vee}\right)$.

Proposition 1.6. (i) ([16], [17]) For $\chi$ unramified,

$$
Z\left(s, \chi, \phi^{0}, \Phi^{0}\right)=\frac{1}{b_{2 n}(s, \chi)} \cdot\left(1-\chi q^{-s-\frac{1}{2}}\right)^{-1} \prod_{j=1}^{n}\left(1-\chi q^{\lambda_{j}-s-\frac{1}{2}}\right)^{-1}\left(1-\chi q^{-\lambda_{j}-s-\frac{1}{2}}\right)^{-1},
$$

where $\chi=\chi(\varpi)$ and

$$
b_{2 n}(s, \chi)=L\left(s+n+\frac{1}{2}, \chi\right) \cdot \prod_{j=1}^{n} L\left(2 s-2 j+2 n+1, \chi^{2}\right),
$$

for $L(s, \chi)=\left(1-\chi \cdot q^{-s}\right)^{-1}$.

(ii) For any matrix coefficient $\phi$ of $\pi^{\vee}$ and any standard section $\Phi(s)$, the product

$$
\prod_{j=1}^{n}\left(1-\chi q^{\lambda_{j}-s-\frac{1}{2}}\right)\left(1-\chi q^{-\lambda_{j}-s-\frac{1}{2}}\right) \cdot Z(s, \chi, \phi, \Phi)
$$

is an entire function of $s$.

The proof of part (ii) of this result, which is rather long, will be given in section 5 .

\section{Proof of Theorem 1.1}

We now turn to the particular case $\pi=\mathbb{1}$. Throughout our discussion, we may assume that either $\chi=1$ or $\chi$ is ramified, since any unramified $\chi$ could be 
absorbed into $s$ via a shift. To avoid confusion, we frequently write $\chi_{0}$ rather than 1 for the trivial character.

The ramified case, in which $\chi \neq 1$, is easy to handle.

Proposition 2.1. If $\chi$ is ramified, then $\mathbb{1}$ does not occur on the boundary for any value of $s$. Moreover, for any standard section $\Phi(s)$, the zeta integral $Z(s, \chi, 1, \Phi)$ is entire and

$$
\operatorname{Hom}_{G \times G}\left(I_{2 n}(s, \chi), \mathbb{1}\right)=\mathbb{C} \cdot Z(s, \chi, 1, \cdot)
$$

for all $s$.

Proof. Applying the criterion of Lemma 1.5 for $\pi=\mathbb{1}$, we find

$$
\begin{aligned}
& \operatorname{dim} \operatorname{Hom}_{G \times G}\left(Q_{r}(s, \chi), \mathbb{1}\right) \\
& =\operatorname{dim}_{\operatorname{Hom}_{r} \times M_{r}}\left(\mathbb{1}, \chi^{-1}||^{-s+n-r+\frac{1}{2}} \otimes \chi^{-1}||^{-s+n-r+\frac{1}{2}} \otimes C^{\infty}\left(G_{n-r}\right)\right) \\
& =\operatorname{dim} \operatorname{Hom}_{\mathrm{GL}_{r} \times \mathrm{GL}_{r}}\left(\mathbb{1}, \chi^{-1}||^{-s+n-r+\frac{1}{2}} \otimes \chi^{-1}||^{-s+n-r+\frac{1}{2}}\right) .
\end{aligned}
$$

This is zero for all $s$ if $\chi$ is ramified. Note that, if $\chi=\chi_{0}$, then this dimension is 1 if and only if $s=n-r+\frac{1}{2}$ (modulo $\frac{2 \pi i}{\log q} \mathbb{Z}$, of course). This fact will be used below.

Now consider the Laurent expansion of the zeta integral at a point $s=s_{0}$, on the space of standard sections:

$$
Z(s, \chi, 1, \Phi)=\frac{\tau_{-k}(\chi, \Phi)}{\left(s-s_{0}\right)^{k}}+\cdots+\tau_{0}(\chi, \Phi)+\ldots
$$

Note that, as mentioned above, the leading term defines a $G \times G$-invariant linear functional

$$
\tau_{-k}: I_{2 n}\left(s_{0}, \chi\right) \longrightarrow \mathbb{C} .
$$

If $k>0$, so that the doubling zeta integral actually has a pole at $s_{0}$, then this functional restricts to zero on the subspace $I_{2 n}\left(s_{0}, \chi\right)^{(0)}$, since the zeta integral is entire for sections in this subspace. It must then give rise to a non-zero element of $\operatorname{Hom}_{G \times G}\left(Q_{r}\left(s_{0}, \chi\right), \mathbb{1}\right)$, for some $r>0$. This contradicts the vanishing of these spaces, so that no pole can occur. The same argument shows that any non-zero element of $\operatorname{Hom}_{G \times G}\left(I_{2 n}\left(s_{0}, \chi\right), \mathbb{1}\right)$ must have non-zero restriction to $I_{2 n}\left(s_{0}, \chi\right)^{(0)}$. Since the restriction of $Z\left(s_{0}, \chi, 1\right)$ to $I_{2 n}\left(s_{0}, \chi\right)^{(0)}$ is non-zero, and is hence a basis for $\operatorname{Hom}_{G \times G}\left(I_{2 n}\left(s_{0}, \chi\right)^{(0)}, \mathbb{1}\right)$, we obtain the claimed result.

For the rest of this section, we consider the more difficult case $\chi=\chi_{0}=1$. We first record some useful facts about the zeta integral in this situation. 
Proposition 2.2. Suppose that $\chi=\chi_{0}$ is trivial.

$Z\left(s, \chi_{0}, 1, \Phi^{0}\right)=\frac{1}{b_{2 n}\left(s, \chi_{0}\right)} \cdot\left(1-q^{-s-\frac{1}{2}}\right)^{-1} \prod_{j=1}^{n}\left(1-q^{j-s-\frac{1}{2}}\right)^{-1}\left(1-q^{-j-s-\frac{1}{2}}\right)^{-1}$.

In particular, this function has only simple poles, and these occur at the points

$$
s_{0} \in X(\mathbb{1}):=\left\{\frac{1}{2}, \frac{3}{2}, \ldots, n-\frac{1}{2}\right\} .
$$

(ii) If $s \notin X(\mathbb{1})$, then $\mathbb{1}$ does not occur on the boundary at s, i.e.,

$$
\operatorname{Hom}_{G \times G}\left(Q_{r}\left(s, \chi_{0}\right), \mathbb{1}\right)=0
$$

for $1 \leq r \leq n$.

(iii) Suppose that $s_{0}=n-r_{0}+\frac{1}{2} \in X(\mathbb{1})$. Then

$$
\operatorname{dim} \operatorname{Hom}_{G \times G}\left(Q_{r}\left(s_{0}, \chi_{0}\right), \mathbb{1}\right)= \begin{cases}1 & \text { if } r=r_{0} \\ 0 & \text { otherwise. }\end{cases}
$$

In particular, $\mathbb{1}$ occurs on a unique boundary component when $s_{0} \in X(\mathbb{1})$.

(iv) For any standard section $\Phi(s) \in I_{2 n}(s, \chi)$, the function $Z\left(s, \chi_{0}, 1, \Phi\right)$ has at most simple poles, and these can only occur at the points $s_{0} \in X(\mathbb{1})$.

(v) Finally, if $s \notin X(\mathbb{1})$, then

$$
\operatorname{Hom}_{G \times G}\left(I_{2 n}(s, \chi), \mathbb{1}\right)=\mathbb{C} \cdot Z\left(s, \chi_{0}, 1, \cdot\right) \neq 0 .
$$

Proof. Part (i) is just a specialization of Proposition 1.6 (i), while parts (ii) and (iii) were proved in the course of the proof of Proposition 2.1. The argument about leading terms in that proof also implies that $Z\left(s, \chi_{0}, 1, \cdot\right)$ cannot have a pole at any point $s \notin X(\mathbb{1})$. This together with (ii) of Proposition 1.6 proves (iv). Finally, the leading term arguments from the proof of Proposition 2.1 imply that (v) holds for $s \notin X(\mathbb{1})$.

To complete the proof of Theorem 1.1, we must consider the case $\chi=\chi_{0}$ and $s_{0}=n-r_{0}+\frac{1}{2} \in X(\mathbb{1})$, so that $\mathbb{1}$ occurs on a unique boundary component. The basic idea is to show that the $G \times G$-invariant functional on this boundary component extends to a functional on all of $I_{2 n}\left(s_{0}\right)$, while the invariant functional defined on the space of functions $S(G)$ on the open cell does not extend. The first step is to compose invariant functionals with the projection from $S(H)$ to $I_{2 n}(s, \chi)$. This allows us to view linear functionals on $I_{2 n}(s, \chi)$ as distributions on $S(H)$ with certain invariance properties under $P \times G \times G$. Note that $H$ can be stratified by $P \times G \times G$ orbits. The second step is to consider certain vector valued distributions whose (unipotent) transformation law under $P$ arises from the Laurent expansion of the induced representation transformation law at $s_{0}$. The standard analysis of Jacquet functors allows 
us to show that, at a given critical point $s_{0}$, the space of such distributions is nonzero for a unique boundary component. The vector valued distributions constructed from the Laurent expansion of the pullback of the zeta integral give an explicit basis element for this space. Finally, by expressing the distribution associated to an arbitrary $G \times G$-invariant functional on a boundary component or on the open cell in terms of these explicitly constructed distributions, we obtain the claimed extension/non-extension properties.

We begin by setting up a little more machinery.

For any $s$, there is a surjective map

$$
\operatorname{pr}(s, \chi): S(H) \longrightarrow I_{2 n}(s, \chi),
$$

given by

$$
\operatorname{pr}(s, \chi)(\varphi)(h):=\int_{P} \chi(p)^{-1}|p|^{-s+\rho_{2 n}} \varphi(p h) d_{l} p,
$$

where $d_{l} p$ is a left Haar measure on $P,[\mathbf{1}]$. The kernel of this map is

$$
\left.\operatorname{ker}(\operatorname{pr}(s, \chi))=\left\langle\ell(p) \varphi-\chi(p)^{-1}|p|^{-s+\rho_{2 n}} \cdot \varphi \quad\right| \quad \text { for } p \in P \text { and } \varphi \in S(H)\right\rangle .
$$

Here the right hand side denotes the subspace of $S(H)$ spanned by these functions; note that it depends on $s$. Also, we have

$$
\operatorname{pr}(s, \chi)(\ell(p) \varphi)=\chi(p)^{-1}|p|^{-s+\rho_{2 n}} \cdot \operatorname{pr}(s, \chi)(\varphi),
$$

where $\ell(p) \varphi(h)=\varphi\left(p^{-1} h\right)$. Composing this map with the zeta integral associated to the trivial representation, we define, for $\operatorname{Re}(s)$ sufficiently large,

$$
z(s, \chi, \varphi):=\int_{G} \int_{P} \chi(p)^{-1}|p|^{-s+\rho_{2 n}} \varphi\left(p \delta_{0} i(g, 1)\right) d_{l} p d g .
$$

This integral defines a distribution on $S(H)$ such that, for $p \in P$

$$
z(s, \chi)(\ell(p) \varphi)=\chi(p)^{-1}|p|^{-s+\rho_{2 n}} \cdot z(s, \chi)(\varphi),
$$

and, for $g_{1}$ and $g_{2} \in G$,

$$
z(s, \chi)\left(r\left(g_{1}, g_{2}\right) \varphi\right)=z(s, \chi)(\varphi)
$$

where $r\left(g_{1}, g_{2}\right) \varphi(h)=\varphi\left(h \cdot i\left(g_{1}, g_{2}\right)\right)$.

The results about the doubling integral reviewed above imply that $z(s, \chi)$ has a meromorphic analytic continuation with at most simple poles at the points $s_{0} \in X(\mathbb{1})$, in the case $\chi=\chi_{0}$.

Suppose that $\chi=\chi_{0}$ and write

$$
z\left(s, \chi_{0}\right)(\varphi)=\frac{\mu_{-1}(\varphi)}{\left(s-s_{0}\right)}+\mu_{0}(\varphi)+\ldots
$$


for the Laurent expansion at $s_{0}$. For any $m \geq 1$, let

$$
\tilde{\mu}^{(m)}(\varphi)=\left(\begin{array}{c}
\mu_{m-2}(\varphi) \\
\mu_{m-3}(\varphi) \\
\vdots \\
\mu_{0}(\varphi) \\
\mu_{-1}(\varphi)
\end{array}\right)
$$

so that $\tilde{\mu}^{(m)}$ is a distribution on $H$ valued in $\mathbb{C}^{m}$.

For $x \in F^{\times}$, let

$$
\gamma_{r}(x)=\frac{(\log |x|)^{r}}{r !} .
$$

By writing out the Laurent expansion, at $s_{0}$, of both sides of the identity

$$
z(s, \chi)(\ell(p) \varphi)=\chi(p)^{-1}|p|^{-s_{0}+\rho_{2 n}}\left(|p|^{-\left(s-s_{0}\right)} \cdot z(s, \chi)(\varphi)\right),
$$

we obtain the following transformation law for $\tilde{\mu}^{(m)}$.

Lemma 2.3. For $p \in P$,

$$
\tilde{\mu}^{(m)}(\ell(p) \varphi)=\chi(p)^{-1}|p|^{-s_{0}+\rho_{2 n}} \cdot\left(\begin{array}{ccccc}
1 & \gamma_{1}(p) & \gamma_{2}(p) & \ldots & \gamma_{m-1}(p) \\
0 & 1 & \gamma_{1}(p) & \ldots & \gamma_{m-2}(p) \\
0 & 0 & 1 & \ldots & \gamma_{m-3}(p) \\
\vdots & \vdots & & \ddots & \vdots \\
0 & 0 & 0 & \ldots & 1
\end{array}\right) \cdot \tilde{\mu}^{(m)}(\varphi)
$$

and

$$
\tilde{\mu}^{(m)}\left(r\left(g_{1}, g_{2}\right) \varphi\right)=\tilde{\mu}^{(m)}(\varphi) .
$$

For convenience, we write $\sigma_{m}(p)$ for the matrix on the right hand side of the first identity of Lemma 2.3. For any $s_{0} \in \mathbb{C}$ and $\chi$, let

$$
D^{(m)}\left(s_{0}, \chi\right)=\operatorname{Hom}_{P \times G \times G}\left(S(H), \chi^{-1}||^{-s_{0}+\rho_{2 n}} \sigma_{m} \otimes \mathbb{1} \otimes \mathbb{1}\right),
$$

be the space of $\mathbb{C}^{m}$-valued distributions on $H$ which satisfy:

$$
\eta(\ell(p) \varphi)=\chi(p)^{-1}|p|^{-s_{0}+\rho_{2 n}} \sigma_{m}(p) \cdot \eta(\varphi),
$$

and

$$
\eta\left(r\left(g_{1}, g_{2}\right) \varphi\right)=\eta(\varphi)
$$

for all $p \in P$ and $g_{1}, g_{2} \in G$.

We will only need to consider the case $m=2$ when $s_{0} \in X(\mathbb{1})$ and $\chi=\chi_{0}$. We write

$$
\zeta_{1}=\left(\begin{array}{c}
\mu_{-1} \\
0
\end{array}\right) \quad \text { and } \quad \zeta_{2}=\left(\begin{array}{c}
\mu_{0} \\
\mu_{-1}
\end{array}\right) \in D^{(2)}\left(s_{0}, \chi_{0}\right)
$$


for the two vector valued distributions which arise from the Laurent expansion of the zeta integral.

Again considering the stratification of $H$, we obtain a filtration

$$
S(H)=S(H)^{(n)} \supset \ldots S(H)^{(r)} \supset S(H)^{(0)}=S\left(\Omega_{0}\right),
$$

with successive quotients

$$
S(H)^{(r)} / S(H)^{(r-1)} \simeq S\left(\Omega_{r}\right)
$$

Proposition 2.4. (i) The restriction of $\mu_{0}$ to $S\left(\Omega_{0}\right) \simeq S(P) \otimes S(G)$ gives a basis for the space

$$
\operatorname{Hom}_{P \times G \times G}\left(S\left(\Omega_{0}\right), \chi^{-1}||^{-s_{0}+\rho_{2 n}} \otimes \mathbb{1} \otimes \mathbb{1}\right)=\mathbb{C} \cdot \mu_{0} .
$$

(ii) For $1 \leq r \leq n$, and for $s_{0}=n-r_{0}+\frac{1}{2}$,

$$
\operatorname{dim} \operatorname{Hom}_{P \times G \times G}\left(S\left(\Omega_{r}\right), \chi^{-1}||^{-s_{0}+\rho_{2 n}} \sigma_{2} \otimes \mathbb{1} \otimes \mathbb{1}\right)= \begin{cases}1 & \text { if } r=r_{0}, \\ 0 & \text { otherwise. }\end{cases}
$$

Proof. By (2.1) and (2.2), part (i) just amounts to the uniqueness of the Haar measures.

Next we prove (ii). If $1 \leq r \leq n$, and using unnormalized induction, we have

$$
\begin{aligned}
& \operatorname{Hom}_{P \times G \times G}\left(S\left(\Omega_{r}\right), \chi^{-1}||^{-s_{0}+\rho_{2 n}} \sigma_{2} \otimes \mathbb{1} \otimes \mathbb{1}\right) \\
& \simeq \operatorname{Hom}_{P \times G \times G}\left(i n d_{S t_{r}}^{P \times G \times G}(\mathbb{1}), \chi^{-1}||^{-s_{0}+\rho_{2 n}} \sigma_{2} \otimes \mathbb{1} \otimes \mathbb{1}\right) \\
& \simeq \operatorname{Hom}_{P \times G \times G}\left(\chi||^{s_{0}-\rho_{2 n}} \sigma_{2}^{\vee} \otimes \mathbb{1} \otimes \mathbb{1}, \operatorname{Ind}_{\mathrm{St}_{r}}^{P \times G \times G}\left(||^{-r} \otimes||^{-r} \otimes \mathbb{1}\right)\right) \\
& \simeq \operatorname{Hom}_{\mathrm{St}_{r}}\left(\chi||^{s_{0}-\rho_{2 n}} \sigma_{2}^{\vee} \otimes \mathbb{1} \otimes \mathbb{1},||^{-r} \otimes||^{-r} \otimes \mathbb{1}\right) \\
& \simeq \operatorname{Hom}_{\mathrm{GL}_{r} \times \mathrm{GL}_{r}}\left(||^{s_{0}-\rho_{2 n}+r} \otimes||^{s_{0}-\rho_{2 n}+r}, \sigma_{2}\right) .
\end{aligned}
$$

Here, in the last line, $\sigma_{2}$ denotes the pullback of the two dimensional representation of $P$ via the imbedding of $\mathrm{GL}_{r} \times \mathrm{GL}_{r}$ into that group given in (1.1) above. In particular, for this embedding, the quantity $|p|$ pulls back to $\left|a_{1}\right|\left|a_{2}\right|$. Also note that, in taking a contragradient, we have used the fact that

$$
\frac{\Delta_{\mathrm{St}_{r}}}{\Delta_{P \times G \times G}}=||^{-r} \otimes||^{-r} \otimes \mathbb{1}
$$

on $\mathrm{St}_{r}$. The last space is zero unless $s_{0}=n-r+\frac{1}{2}$, and in that case has dimension 1, as claimed. 
In general, if $\eta \in D^{(2)}\left(s_{0}, \chi_{0}\right)$ restricts to zero on $S(H)^{(r-1)}$, then it induces a homomorphism

$$
\bar{\eta} \in \operatorname{Hom}_{P \times G \times G}\left(S\left(\Omega_{r}\right), \chi^{-1}||^{-s_{0}+\rho_{2 n}} \sigma_{2} \otimes \mathbb{1} \otimes \mathbb{1}\right)
$$

on the quotient of the next step of the filtration. Part (ii) of Proposition 2.4 forces the $\bar{\eta}$ 's to vanish when $r \neq r_{0}$. Note that $\mu_{-1}$ and hence $\zeta_{1}$ vanish on $S\left(\Omega_{0}\right)$, since the zeta integral has no poles for $\varphi$ in this space. On the other hand, $\zeta_{1}$ is not identically zero, since a pole occurs. Thus, its restriction to $S(H)^{\left(r_{0}\right)}$ is non-zero, and the resulting $\bar{\zeta}_{1}$ gives a basis for the space in (ii) of Proposition 2.4. These considerations yield the following result.

Corollary 2.5. Suppose that $s_{0}=n-r_{0}+\frac{1}{2} \in X(\mathbb{1})$.

(i) If $\eta \in D^{(2)}\left(s_{0}, \chi_{0}\right)$ restricts to zero on $S\left(\Omega_{0}\right)$, then $\eta$ is zero on $S(H)^{\left(r_{0}-1\right)}$.

(ii) If $\eta \in D^{(2)}\left(s_{0}, \chi_{0}\right)$ restricts to zero on $S(H)^{\left(r_{0}\right)}$, then $\eta=0$.

(iii) The distribution $\zeta_{1}$ restricts to zero on $S(H)^{\left(r_{0}-1\right)}$, and its restriction to $S(H)^{\left(r_{0}\right)}$ gives a basis for the space

$$
\operatorname{Hom}_{P \times G \times G}\left(S\left(\Omega_{r_{0}}\right), \chi^{-1}||^{-s_{0}+\rho_{2 n}} \sigma_{2} \otimes \mathbb{1} \otimes \mathbb{1}\right)=\mathbb{C} \cdot \bar{\zeta}_{1} .
$$

We now return to the proof of Theorem 1.1.

Now suppose that $s_{0} \in X(\mathbb{1})$ and that $\lambda \in \operatorname{Hom}_{G \times G}\left(I_{2 n}\left(s_{0}, \chi_{0}\right)\right.$, 1$)$. Let

$$
\lambda^{\prime}=\lambda \circ \operatorname{pr}\left(s_{0}, \chi_{0}\right) \in \operatorname{Hom}_{P \times G \times G}\left(S(H),||^{-s_{0}+\rho_{2 n}} \otimes \mathbb{1} \otimes \mathbb{1}\right)
$$

be the pullback of $\lambda$ via the projection map, and let

$$
\lambda^{\prime \prime}=\left(\begin{array}{c}
\lambda^{\prime} \\
0
\end{array}\right) \in D^{(2)}\left(s_{0}, \chi_{0}\right) .
$$

By (i) of Proposition 2.4, there is a constant $c$ such that

$$
\left.\lambda^{\prime}\right|_{S\left(\Omega_{0}\right)}=\left.c \cdot \mu_{0}\right|_{S\left(\Omega_{0}\right)} .
$$

Since the zeta integral has no pole for $\varphi \in S\left(\Omega_{0}\right), \mu_{-1}$ restricts to zero on this space. Thus, passing to elements of $D^{(2)}\left(s_{0}, \chi_{0}\right)$, we have

$$
\left.\left(\lambda^{\prime \prime}-c \cdot \zeta_{2}\right)\right|_{S\left(\Omega_{0}\right)}=0 .
$$

Set $\eta=\lambda^{\prime \prime}-c \cdot \zeta_{2}$. By (i) and (iii) of Corollary 2.5, there is a constant $c^{\prime}$ such that $\eta-c^{\prime} \cdot \zeta_{1}$ restricts to zero on $S(H)^{\left(r_{0}\right)}$, and hence, by (i) of Corollary 2.5, $\eta=c^{\prime} \cdot \zeta_{1}$. But then, the second component, $-c \cdot \mu_{-1}$, of $\eta$, must be identically zero. This implies that $c=0$, and that, in fact,

$$
\lambda=c^{\prime} \cdot \mu_{-1} .
$$

This completes the proof of Theorem 1.1 and yields some extra information.

Corollary 2.6. (i) For all values of $s_{0}$ and $\chi$, the one dimensional space

$$
\operatorname{Hom}_{G \times G}\left(I_{2 n}\left(s_{0}, \chi\right), \mathbb{1}\right)
$$


is spanned by the leading term of the Laurent expansion at $s_{0}$ of the zeta integral $Z(s, \chi, 1)$.

(ii) If $\chi=\chi_{0}$ and $s_{0} \in X(\mathbb{1})$, then the space

$$
\operatorname{Hom}_{P \times G \times G}\left(S(H), \chi^{-1}||^{-s_{0}+\rho_{2 n}} \sigma_{2} \otimes \mathbb{1} \otimes \mathbb{1}\right)
$$

has dimension 2 and is spanned by the Laurent vectors $\zeta_{1}$ and $\zeta_{2}$ defined using the first two terms of the Laurent expansion of the zeta integral $z\left(s, \chi_{0}\right)$ at $s_{0}$.

\section{The local theta correspondence}

In this section, we prove some basic results about 'early' and 'late' occurrence in the theta correspondence, for dual pairs of type $(S p, O)$. We consider both the symplectic and metaplectic case. We fix, once and for all, a nontrivial additive character $\psi$ of $F$ which we assume to be trivial on $\mathcal{O}_{F}$ and non-trivial on $\mathcal{P}_{F}^{-1}$, where $\mathcal{P}_{F}=\varpi \mathcal{O}_{F}$ is the maximal ideal of $\mathcal{O}_{F}$.

Let $V,($,$) be a vector space of dimension m$ over $F$ with a non-degenerate symmetric bilinear form. Let

$$
G^{\prime}=G_{n}^{\prime}= \begin{cases}M p(W) & \text { if } m \text { is odd } \\ S p(W) & \text { if } m \text { is even }\end{cases}
$$

where $W=W_{n}$ is a symplectic vector space of dimension $2 n$, as in section 1 . Here $M p(W)$ denotes the metaplectic extension of $S p(W)$, which we take to be the extension

$$
1 \longrightarrow \mathbb{C}^{1} \longrightarrow M p(W) \longrightarrow S p(W) \longrightarrow 1,
$$

obtained from the usual 2 -fold covering via the inclusion of $\{ \pm 1\}$ into $\mathbb{C}^{1}$.

In the symplectic group $S p\left(V \otimes_{F} W\right)$ of the vector space $V \otimes_{F} W$, of dimension $2 m n$ over $F$ with symplectic form $(,) \otimes<,>$, we then have the usual reductive dual pair $(O(V), S p(W))$. The homomorphism

$$
i: O(V) \times S p(W) \longrightarrow S p\left(V \otimes_{F} W\right)
$$

has a lifting to a homomorphism

$$
\tilde{i}: O(V) \times G^{\prime} \longrightarrow \mathbb{G}^{\prime},
$$

where

$$
\mathbb{G}^{\prime}= \begin{cases}M p\left(V \otimes_{F} W\right) & \text { if } m \text { is odd } \\ S p\left(V \otimes_{F} W\right) & \text { if } m \text { is even. }\end{cases}
$$

The pullback to $G^{\prime} \times O(V)$ of the Weil representation $\omega_{\psi}$ of $\mathbb{G}^{\prime}$, yields a representation of this product group on the space $S\left(V^{n}\right)$, in the standard 
Schrödinger model. The lifting $\tilde{i}$ is unique provided we require that $h \in O(V)$ acts by $\omega(h) \varphi(x)=\varphi\left(h^{-1} x\right)$, and that, when $m$ is odd, the central $\mathbb{C}^{1}$ in $G^{\prime}$ acts in $S\left(V^{n}\right)$ by $\omega(z) \varphi=z \cdot \varphi$.

If $\pi$ is an irreducible admissible representation of $G^{\prime}$, we let $S(\pi)$ be the maximal quotient of $S=S\left(V^{n}\right)$ on which $G^{\prime}$ acts by a multiple of $\pi$, and we write

$$
S(\pi) \simeq \pi \otimes \Theta(\pi, V) .
$$

The Howe duality principle is the following.

Theorem 3.1. (Howe, [4], Waldspurger, [22]) Assume that the residue characteristic of $F$ is odd.

(i) $\Theta(\pi, V)$ is a finitely generated representation of $O(V)$.

(ii) If $S(\pi) \neq 0$, then $\Theta(\pi, V)$ has a unique irreducible quotient $\theta(\pi, V)$.

(iii) The correspondence $\pi \mapsto \theta(\pi, V)$ defines a bijection between the subsets of $\operatorname{Irr}\left(G^{\prime}\right)$ and $\operatorname{Irr}(O(V))$ which occur in the correspondence.

This duality principle is also expected to hold when the residue characteristic of $F$ is even. Note that the space $\Theta(\pi, V)$ is defined, in any case. As in [3], we will make use of only the following weak version of (i):

Proposition 3.2. For any irreducible admissible representation $\pi$ of $G^{\prime}$ the space $\Theta(\pi, V)$ is either 0 , or, as a representation of $O(V)$, it has finite length and hence has an irreducible admissible quotient.

Note that there is no restriction on the residue characteristic here. This result is proved in Chapitre 3, Theorem 4, 2) a), p. 69 of [13], via the methods in [18] and [5].

We now consider the behavior of the correspondence with respect to Witt towers. For any integer $r \geq 1$, let $V_{r, r}=F^{2 r}$ with inner product given by the matrix $\left(\begin{array}{cc} & 1_{r} \\ 1_{r} & \end{array}\right)$. Two quadratic spaces $V$ and $V^{\prime}$ are said to lie in the same Witt tower if, up to isomorphism, they differ by a $V_{r, r}$, e.g., if $V^{\prime} \simeq V+V_{r, r}$. The following facts are well known.

Lemma 3.3. (i) (persistence) For any irreducible admissible representation $\pi$ of $G^{\prime}$, and for any $r \geq 1$,

$$
\Theta(\pi, V) \neq 0 \Longrightarrow \Theta\left(\pi, V+V_{r, r}\right) \neq 0
$$

(ii) (stable range (Howe)) If $V=V_{0}+V_{r, r}$ with $r \geq 2 n$, then, for all irreducible admissible representations $\pi$ of $G^{\prime}$

$$
\Theta(\pi, V) \neq 0,
$$


provided, in the case $m=\operatorname{dim} V$ is odd, $\pi$ is assumed to be genuine.

The isomorphism class of $V$ is determined by the invariants $m=\operatorname{dim}_{F} V$, the quadratic character $\chi_{V}$ of $F^{\times}$defined by

$$
\chi_{V}(x)=\left(x,(-1)^{\frac{m(m-1)}{2}} \operatorname{det} V\right),
$$

and the Hasse invariant $\epsilon(V)= \pm 1$. We normalize our Hasse invariant so that it is constant on Witt towers. Note that the character $\chi_{V}$ also has this property, so that a Witt tower is determined by a choice of $\chi=\chi_{V}$ and $\epsilon=\epsilon(V)$. More precisely, the Witt towers can then be described as follows, [18].

For $m$ even and $\chi=1$, we have the split tower, $\left\{V_{m}^{+}=V_{r, r}\right\}$, with $m=$ $2 r$. Here we view the first element of the tower to be the 0 dimensional space $V_{0,0}(!)$. Let $B$ be the division quaternion algebra over $F$, and let $V_{4}^{-}=B$, with $(x, y)=\operatorname{tr}\left(x y^{\iota}\right)$, where $\iota$ is the main involution of $B$. Then the quaternionic tower is $\left\{V_{m}^{-}\right\}, m=2 r \geq 4$, where $V_{m}^{-}=V_{4}^{-}+V_{r-2, r-2}$.

For $m$ even and $\chi \neq 1$, we let $k$ be the quadratic extension of $F$ corresponding to the character $\chi$, and let $V_{2}^{+}=k$ with $(x, y)=\operatorname{tr}\left(x y^{\iota}\right)$, where $\iota$ is the non-trivial Galois automorphism of $k$ over $F$. Let $V_{2}^{-}=k$ with $(x, y)=\kappa \cdot \operatorname{tr}\left(x y^{\iota}\right)$, where $\chi(\kappa)=-1$. Then let $V_{2 r}^{ \pm}=V_{2}^{ \pm}+V_{r-1, r-1}$, so that $\left\{V_{m}^{+}\right\}$and $\left\{V_{m}^{-}\right\}, m \geq 2$ are the two tower associated to $\chi$.

For $m=2 r+1$ odd, and for a fixed quadratic character $\chi(x)=(x, \alpha)_{F}$, where $(,)_{F}$ is the quadratic Hilbert symbol for $F$, let $V_{1}^{+}=F$ with $(x, y)=$ $\alpha \cdot x y$. Let $B$ be as before, and let $V_{3}^{-}=\{x \in B \mid \operatorname{tr}(x)=0\}$. Here we set $(x, y)=\alpha^{\prime} \cdot \operatorname{tr}(x y)$, where the scalar $\alpha^{\prime} \in F^{\times}$is chosen so that $\chi_{V_{3}^{-}}=\chi$. Then our Witt towers associated to $\chi$ are $\left\{V_{m}^{+}\right\}, m=2 r+1 \geq 1$, with $V_{m}^{+}=V_{1}^{+}+V_{r, r}$ and $\left\{V_{m}^{-}\right\}, m=2 r+1 \geq 3$, with $V_{m}^{-}=V_{3}^{-}+V_{r-1, r-1}$.

Note that, in all cases, the Hasse invariant is given by $\epsilon\left(V_{m}^{ \pm}\right)= \pm 1$.

Definition 3.4. Fix a quadratic character $\chi$ and a parity ${ }^{3}$. Then, for any irreducible admissible representation $\pi$ of $G^{\prime}$, let $m_{\chi}^{ \pm}(\pi)$ be the smallest integer $m$, of the fixed parity, such that $\Theta_{\chi}\left(\pi, V_{m}^{ \pm}\right) \neq 0$. In the case of odd parity, $\pi$ is assumed to be genuine.

Part (iii) of Lemma 3.1, i.e., the stable range condition, immediately yields the following.

Lemma 3.5. (i) For $\chi=\chi_{0}=1$ and even parity,

$$
0 \leq m_{\chi_{0}}^{+}(\pi) \leq 4 n, \quad \text { and } \quad 4 \leq m_{\chi_{0}}^{-}(\pi) \leq 4 n+4
$$

\footnotetext{
${ }^{3}$ By this we mean that we consider quadratic spaces of dimension $m$ where the parity of $m$ is fixed.
} 
(ii) For $\chi \neq 1$ and even parity, $2 \leq m_{\chi}^{ \pm}(\pi) \leq 4 n+2$.

(iii) For odd parity,

$$
1 \leq m_{\chi}^{+}(\pi) \leq 4 n+1, \quad \text { and } \quad 3 \leq m_{\chi}^{-}(\pi) \leq 4 n+3 .
$$

In fact, these first occurrence indices should be linked by the following conservation relation (Conjecture $\mathrm{A}$, of the introduction):

Conjecture 3.6. Fix a parity and a quadratic character $\chi$. Then, for all irreducible admissible representations $\pi$ of $G^{\prime}$,

$$
m_{\chi}^{+}(\pi)+m_{\chi}^{-}(\pi)=4 n+4 .
$$

Here, in the case of odd parity, $\pi$ is assumed to be genuine.

In effect, if a representation $\pi$ occurs 'early' in one tower, it must occur 'late' in the other! For example, in the case of even parity, $\pi$ occurs 'early' if $\Theta(\pi, V) \neq 0$ for $\operatorname{dim} V=2 n+2-2 r \leq 2 n$. For $V^{\prime}$ in the other tower, the conjectured relation then requires that $\Theta\left(\pi, V^{\prime}\right)=0$ if $\operatorname{dim} V^{\prime} \leq 2 n+2 r$. In the case of odd parity, the conjectured relation implies theta dichotomy (Conjecture B of the introduction).

Conjecture 3.7. Fix a quadratic character $\chi$. For any irreducible admissible genuine representation $\pi$ of $G^{\prime}$, precisely one of the spaces $\Theta_{\chi}\left(\pi, V_{2 n+1}^{+}\right)$and $\Theta_{\chi}\left(\pi, V_{2 n+1}^{-}\right)$is non-zero.

Here the subscript $\chi$ serves as a reminder that we are considering the pair of Witt towers associated to $\chi$.

The analogous conjectures for orthogonal groups are mentioned in the introduction. In the case of unitary groups, the conjectures appear in section 7 of $[3]$.

Let $H=S p(\mathbb{W})$ be as in section 1, where $\mathbb{W}=W+W_{-}$, and let

$$
H^{\prime}= \begin{cases}S p(\mathbb{W}) & \text { in the even parity case } \\ M p(\mathbb{W}) & \text { in the odd parity case. }\end{cases}
$$

The map $\iota: G \times G \longrightarrow H$ of section 1 yields a map

$$
\iota^{\prime}: G^{\prime} \times G^{\prime} \longrightarrow H^{\prime},
$$

which is unique if we require that, in the case of odd parity,

$$
\iota^{\prime}: \mathbb{C}^{1} \times \mathbb{C}^{1} \longrightarrow \mathbb{C}^{1}, \quad \iota^{\prime}\left(z_{1}, z_{2}\right)=z_{1} z_{2}^{-1} .
$$

In the case of even parity, we let $I_{2 n}^{\prime}(s, \chi)$ be the degenerate principal series representation $I_{2 n}(s, \chi)$ of $H^{\prime}=H$, as in section 1 . In the case of odd parity, we note that the restriction of the extension $H^{\prime}$ to the Levi factor of the Siegel 
parabolic $P$ is given by

$$
M^{\prime} \simeq M \times \mathbb{C}^{1}
$$

with multiplication

$$
\left(m\left(a_{1}\right), z_{1}\right) \cdot\left(m\left(a_{2}\right), z_{2}\right)=\left(m\left(a_{1} a_{2}\right), z_{1} z_{2}\left(\operatorname{det} a_{1}, \operatorname{det} a_{2}\right)_{F}\right) .
$$

Here we use the conventions of [6]. For a character $\chi$ of $F^{\times}$, we set

$$
\chi^{\psi}(x)=\chi(x) \gamma_{F}\left(x, \frac{1}{2} \psi\right)^{-1},
$$

where $\gamma_{F}\left(x, \frac{1}{2} \psi\right)$ is in [19],[6]. We then define a (genuine) character $\chi^{\psi}$ of $M^{\prime}$ by

$$
\chi^{\psi}((m(a), z))=z \cdot \chi^{\psi}(\operatorname{det} a) .
$$

We extend this to $P^{\prime}=M^{\prime} N$, trivially on $N$, and let

$$
I_{2 n}^{\prime}(s, \chi)=I_{P^{\prime}}^{H^{\prime}}\left(\chi^{\psi}||^{s}\right),
$$

be the (genuine) degenerate principal series of $H^{\prime}$. In both cases, we restrict the representation $I_{2 n}^{\prime}(s, \chi)$ to $G^{\prime} \times G^{\prime}$ via $\iota^{\prime}$.

First, we extend Conjecture 1.2 to the metaplectic case:

Conjecture 1.2' For all irreducible admissible (genuine) representations $\pi$ of $G^{\prime}$, and for all $s$ and $\chi$,

$$
\operatorname{dim} \operatorname{Hom}_{G^{\prime} \times G^{\prime}}\left(I_{2 n}^{\prime}(s, \chi), \pi \otimes \pi^{\vee}\right)=1 .
$$

Our main results are the following:

Theorem 3.8. Fix a quadratic character $\chi$ and a parity. Then for any irreducible admissible (genuine) representation $\pi$ of $G^{\prime}$,

$$
m_{\chi}^{+}(\pi)+m_{\chi}^{-}(\pi) \geq 4 n+4 .
$$

Theorem 3.9. Fix a quadratic character $\chi$ and a parity. Suppose that $\pi$ is an irreducible admissible representation of $G^{\prime}$ such that

$$
\operatorname{dim} \operatorname{Hom}_{G_{n} \times G_{n}}\left(I_{2 n}\left(s_{0}, \chi\right), \pi \otimes \pi^{\vee}\right)=1,
$$

for all $s_{0}$ in the set:

$$
\text { Crit }= \begin{cases}\left\{-n-\frac{1}{2},-n+\frac{1}{2}, \ldots, n-\frac{1}{2}, n+\frac{1}{2}\right\} & \text { for even parity and } \chi=1, \\ \left\{-n+\frac{1}{2}, \ldots, n-\frac{1}{2}\right\} & \text { for even parity and } \chi \neq 1, \\ \{-n,-n+1, \ldots, n-1, n\} & \text { for odd parity. }\end{cases}
$$

Then

$$
m_{\chi}^{+}(\pi)+m_{\chi}^{-}(\pi)=4 n+4
$$


In particular, Conjectures 3.6 and 3.7 hold for any supercuspidal representation $\pi$.

Of course, Theorem 3.9 together with Conjectures 1.2 and $1.2^{\prime}$ imply Conjecture 3.6 and 3.7 for all $\pi$.

Corollary 3.10. For the trivial representation $\mathbb{1}$ of $G^{\prime}$, and for even parity, if $\chi=\chi_{0}=1$,

$$
m_{\chi_{0}}^{+}(\mathbb{1})=0 \quad \text { and } \quad m_{\chi_{0}}^{-}(\mathbb{1})=4 n+4,
$$

while, if $\chi \neq 1$,

$$
m_{\chi}^{+}(\mathbb{1})=m_{\chi}^{-}(\mathbb{1})=2 n+2 .
$$

In particular, $\Theta_{\chi_{0}}\left(\mathbb{1}, V_{4 n+2}^{-}\right)=0$.

Note that Conjecture 3.6 implies that $\mathbb{1}$ is the only irreducible admissible representation of $G^{\prime}$ for which $\Theta_{\chi_{0}}\left(\pi, V_{4 n+2}^{-}\right)=0$, and that any representation $\pi \neq \mathbb{1}$ for which the conservation principle holds must have $\Theta_{\chi_{0}}\left(\pi, V_{4 n+2}^{-}\right) \neq 0$.

More precise information about first occurrence can be given in certain cases. In the case of even parity with $\chi \neq 1$, choose $\alpha \in F^{\times}$such that $\chi(\alpha)=-1$. For a representation $\sigma$ of $G^{\prime}=G=S p(W)$, let $\delta_{\alpha}(\sigma)(g)=\sigma\left(g^{\alpha}\right)$, where

$$
g^{\alpha}=\left(\begin{array}{ll}
1 & \\
& \alpha
\end{array}\right) g\left(\begin{array}{ll}
1 & \\
& \alpha
\end{array}\right)^{-1} .
$$

Note that, if $\sigma=\omega_{V_{m}^{\epsilon}}$ is the Weil representation of $G$ determined by the space $V_{m}^{\epsilon}$ of character $\chi$, then $\delta_{\alpha}(\sigma) \simeq \omega_{V_{m}^{-\epsilon}}$ is the Weil representation defined by $V_{m}^{-\epsilon}$. Thus, for any irreducible admissible representation $\pi$, we have

$$
m_{\chi}^{\epsilon}(\pi)=m_{\chi}^{-\epsilon}\left(\delta_{\alpha}(\pi)\right)
$$

Corollary 3.11. In the case of even parity with $\chi \neq 1$, suppose that $\delta_{\alpha}(\pi) \simeq$ $\pi$. Then $m_{\chi}^{+}(\pi)=m_{\chi}^{-}(\pi) \geq 2 n+2$. Moreover, if $\pi$ satisfies the hypothesis of Theorem 3.9, then

$$
m_{\chi}^{+}(\pi)=m_{\chi}^{-}(\pi)=2 n+2 .
$$

For example, $m_{\chi}^{ \pm}(\mathbb{1})=2 n+2$.

\section{Proofs of Theorems 3.8 and 3.9}

Before proving these results, we need a few more preliminaries. 
For any quadratic space $V$, let

$$
R_{2 n}(V)=S\left(V^{2 n}\right)_{O(V)}
$$

be the maximal quotient of $S\left(V^{2 n}\right)$ on which $O(V)$ acts trivially, and note that this is a representation of $H^{\prime}$. Recall that there is a natural $H^{\prime}$ equivariant map

$$
\lambda: S\left(V^{2 n}\right) \longrightarrow I_{2 n}^{\prime}\left(s_{0}, \chi\right), \quad \varphi \mapsto(h \mapsto(\omega(h) \varphi)(0)),
$$

where $s_{0}=\frac{m}{2}-\rho_{2 n}$ and $\chi=\chi_{V}$. Moreover, this map induces an inclusion, [18],

$$
R_{2 n}(V) \hookrightarrow I_{2 n}^{\prime}\left(s_{0}, \chi\right) .
$$

A complete account of the reducibility of the degenerate principal series $I_{2 n}^{\prime}(s, \chi)$ in terms of these submodules can be found in [2], [9], [8], when $m=\operatorname{dim} V$ is even, and in [20] when $m$ is odd. These results will be briefly reviewed below.

The following result is, by now, quite standard [4], [18], [3], and its proof is just like that of Proposition 3.1 of [3].

Proposition 4.1. Let $\pi$ be an irreducible admissible representation of $G^{\prime}$. Then

$$
\Theta(\pi, V) \neq 0 \Longleftrightarrow \operatorname{Hom}_{G^{\prime} \times G^{\prime}}\left(R_{2 n}(V), \pi \otimes \pi^{\vee}\right) \neq 0 .
$$

We now begin the proof of Theorems 3.8 and 3.9 by proving the first part of Corollary 3.10:

Lemma 4.2. For even parity and for $\chi=\chi_{0}=1$,

$$
\operatorname{Hom}_{G^{\prime} \times G^{\prime}}\left(R_{2 n}\left(V_{m}^{-}\right), \mathbb{1} \otimes \mathbb{1}\right)=0,
$$

for $m \leq 4 n+2$, and

$$
\operatorname{dim} \operatorname{Hom}_{G^{\prime} \times G^{\prime}}\left(R_{2 n}\left(V_{4 n+4}^{-}\right), \mathbb{1} \otimes \mathbb{1}\right)=1 .
$$

Thus, the trivial representation does not occur in the quaternionic tower until the stable range, and

$$
m_{\chi_{0}}^{+}(\mathbb{1})+m_{\chi_{0}}^{-}(\mathbb{1})=4 n+4 .
$$

Proof. First observe that we view the trivial representation as the Weil representation associated to the space $V_{0,0}$ at the base of the split tower. Thus, $\Theta\left(\mathbb{1}, V_{0,0}\right) \neq 0$ (formally), and so, by (i) of Lemma 3.3 together with Lemma 4.1, we have

$$
\operatorname{Hom}_{G^{\prime} \times G^{\prime}}\left(R_{2 n}\left(V_{m}^{+}\right), \mathbb{1} \otimes \mathbb{1}\right) \neq 0,
$$


for $m=0,2, \ldots$ in the split tower. Moreover, for $m \geq 4 n+4$ even, we have, $[9]$

$$
R_{2 n}\left(V_{m}^{+}\right)=R_{2 n}\left(V_{m}^{-}\right)=I_{2 n}\left(s_{0}, \chi_{0}\right),
$$

so that the combination of Theorem 1.1 and Proposition 4.1 yield the nonvanishing assertion. One could also invoke (ii) of Lemma 3.3. Also, by (i) of Lemma 3.3 , to prove that $m_{\chi_{0}}^{-}(\mathbb{1})=4 n+4$, it suffices to show that the space

$$
\operatorname{Hom}_{G^{\prime} \times G^{\prime}}\left(R_{2 n}\left(V_{4 n+2}^{-}\right), \mathbb{1} \otimes \mathbb{1}\right)
$$

is zero. But, as shown in [2] and [9], $R_{2 n}\left(V_{4 n+2}^{-}\right)$is the unique irreducible submodule of $I_{2 n}^{\prime}\left(\rho_{2 n}, \chi_{0}\right)$ and is precisely the image of the normalized intertwining operator

$$
M_{2 n}^{*}\left(-\rho_{2 n}, \chi_{0}\right): I_{2 n}\left(-\rho_{2 n}, \chi_{0}\right) \longrightarrow I_{2 n}\left(\rho_{2 n}, \chi_{0}\right) .
$$

If the space (4.1) were non-zero, we would obtain a non-zero element of the space

$$
\operatorname{Hom}_{G^{\prime} \times G^{\prime}}\left(I_{2 n}\left(-\rho_{2 n}, \chi_{0}\right), \mathbb{1} \otimes \mathbb{1}\right)
$$

by composing with the intertwining operator (4.2). This element would be trivial on the vector $\Phi^{0}\left(-\rho_{2 n}\right)$, which is, in fact, the constant function 1 and spans the kernel of $M_{2 n}^{*}\left(-\rho_{2 n}, \chi_{0}\right)$. But since $-\rho_{2 n} \notin X(\mathbb{1})$, (v) and (i) of Proposition 2.2 imply that the zeta integral $Z\left(-\rho_{2 n}, \chi_{0}, 1\right)$ spans the space (4.3) and does not vanish on $\Phi^{0}\left(-\rho_{2 n}\right)$. This proves that (4.1) must be zero, as required.

Proof of Theorem 3.8. Fix $\chi$ and a parity, and suppose that $\pi$ is an irreducible admissible representation of $G^{\prime}$, which is genuine in the case of odd parity. Suppose that $\Theta\left(\pi, V_{a}^{+}\right) \neq 0$ and $\Theta\left(\pi, V_{b}^{-}\right) \neq 0$, where $V_{a}^{+}$and $V_{b}^{-}$are spaces of character $\chi$, dimensions $a$ and $b$, respectively, and Hasse invariants +1 and -1. There are then non-zero $G^{\prime}$-intertwining maps

$$
\lambda: S\left(\left(V_{a}^{+}\right)^{n}\right) \longrightarrow \pi \quad \text { and } \quad \mu: S\left(\left(V_{b}^{-}\right)^{n}\right) \longrightarrow \pi .
$$

Recall that the MVW involution [13],[3] on the set of smooth representations of $G^{\prime}$ is defined by set $\tau^{c}(g)=\tau\left(g^{c}\right)$, where, in the case of even parity, $g^{c}=$ $c g c^{-1}$, for $c=\left(\begin{array}{cc}1_{n} & 0 \\ 0 & -1_{n}\end{array}\right)$. In the case of odd parity, we use the same notation to denote the unique lift of this automorphism to $G^{\prime}$ whose restriction to the central $\mathbb{C}^{1}$ is given by $z \mapsto z^{-1}$, [3]. Also recall that, if $\tau$ is irreducible, then $\tau^{c} \simeq \tau^{\vee}$, the contragredient of $\tau$. We apply this involution to obtain an intertwining map

$$
\mu^{c}: S\left(\left(V_{b}^{-}\right)^{n}\right)^{c} \longrightarrow \pi^{\vee}
$$


On the other hand, the representation of $G^{\prime}$ on $S\left(\left(V_{b}^{-}\right)^{n}\right)^{c}$ is isomorphic to the complex conjugate of its representation on $S\left(\left(V_{b}^{-}\right)^{n}\right)$. Note that these representations are preunitary. We thus obtain a nonzero intertwining map

$$
\lambda \otimes \mu^{c} \in \operatorname{Hom}_{G^{\prime} \times G^{\prime}}\left(S\left(\left(V_{a}^{+}\right)^{n}\right) \otimes S\left(\left(V_{b}^{-}\right)^{n}\right)^{c}, \pi \otimes \pi^{\vee}\right) .
$$

We next restrict these representations to the diagonal $G^{\prime}$ in $G^{\prime} \times G^{\prime}$. Since the central $\mathbb{C}^{1}$ now acts trivially, we obtain representations of $G=S p(W)$. Moreover, the representation of $G$ on $S\left(\left(V_{a}^{+}\right)^{n}\right) \otimes S\left(\left(V_{b}^{-}\right)^{n}\right)^{c}$ is isomorphic to the Weil representation of this group on $S\left(\left(V_{a}^{+}+\left(-V_{b}^{-}\right)\right)^{n}\right)$, where $-V_{b}^{-}$is the space $V_{b}^{-}$with the negative of its quadratic form. Thus, we obtain a nonzero intertwining map in

$$
\operatorname{Hom}_{G}\left(S\left(\left(V_{a}^{+}+\left(-V_{b}^{-}\right)\right)^{n}\right), \mathbb{1}\right),
$$

by composing with the invariant pairing $\pi \otimes \pi^{\vee} \longrightarrow \mathbb{1}$. The following fact is easily checked:

Lemma 4.3. The space $V_{a}^{+}+\left(-V_{b}^{-}\right)$has trivial character, i.e., square discriminant, and Hasse invariant -1. Specifically, it is the quaternionic space of dimension $a+b$.

But now, we have $\Theta\left(\mathbb{1}, V_{a}^{+}+\left(-V_{b}^{-}\right)\right) \neq 0$, so that, by Lemma $4.2, a+b \geq$ $4 n+4$, as claimed.

Proof of Theorem 3.9. We now must make use of the way in which the representations $R_{2 n}(V)$ sit inside of $I_{2 n}^{\prime}\left(s_{0}, \chi\right)$, where $\chi=\chi_{V}$ and $s_{0}=\frac{m}{2}-\frac{n+1}{2}$. Here $\chi$ will be fixed throughout our argument. This structure can be summarized as follows, [2], [9], [20], [21]. First of all, we recall that $I_{2 n}^{\prime}(s, \chi)$ is irreducible unless

$$
s=s_{0} \in \begin{cases}\left\{-n-\frac{1}{2},-n+\frac{1}{2}, \ldots, n-\frac{1}{2}, n+\frac{1}{2}\right\} & \text { for even parity and } \chi=\chi_{0}=1, \\ \left\{-n+\frac{1}{2}, \ldots, n-\frac{1}{2}\right\} & \text { for even parity and } \chi \neq 1, \\ \{-n,-n+1, \ldots, n-1, n\} & \text { for odd parity. }\end{cases}
$$

Moreover, there are 3 constituents, except in the cases

$s=s_{0}= \begin{cases}\left\{-n-\frac{1}{2},-n+\frac{1}{2}, n-\frac{1}{2}, n+\frac{1}{2}\right\} & \text { for even parity and } \chi=\chi_{0}=1, \\ \{-n, n\} & \text { for odd parity, }\end{cases}$

when the composition series has length 2 . The constituents can be described in terms of the $R_{2 n}(V)$ 's. We say that the spaces $V_{m_{0}}^{+}$and $V_{m}^{+}$(resp. $V_{m_{0}}^{-}$and $V_{m}^{-}$), with $0 \leq m_{0} \leq m$, are complementary if $m+m_{0}=4 n+2$. In this case, note that

$$
s_{0}=\frac{m}{2}-\frac{2 n+1}{2} \quad \text { and } \quad-s_{0}=\frac{m_{0}}{2}-\frac{2 n+1}{2} .
$$


Note that certain values of the parameters $\chi$ and $m_{0}$ are excluded. Specifically, the spaces $V_{0}^{-}$and $V_{1}^{-}$for any $\chi, V_{2}^{-}$for $\chi=\chi_{0}$, and $V_{0}^{+}$for $\chi \neq 1$ do not exist. In these cases, we formally take $R_{2 n}\left(V_{m_{0}}^{ \pm}\right)=0$. Similarly, if $m_{0}=0$, we take $R_{2 n}\left(V_{0}^{+}\right)$to be the trivial representation, which is the unique irreducible submodule of $I_{2 n}\left(-n-\frac{1}{2}, \chi_{0}\right)$ in the case of even parity. If $0 \leq m_{0} \leq 2 n+1$, then $R_{2 n}\left(V_{m_{0}}^{ \pm}\right)$is irreducible, provided the space $V_{m_{0}}^{ \pm}$exists. As submodules of $I_{2 n}^{\prime}\left(-s_{0}, \chi\right)$,

$$
R_{2 n}\left(V_{m_{0}}^{+}\right) \cap R_{2 n}\left(V_{m_{0}}^{-}\right)=0 .
$$

When $m_{0}=m=2 n+1$, we have [21]

$$
R_{2 n}\left(V_{m_{0}}^{+}\right) \oplus R_{2 n}\left(V_{m_{0}}^{-}\right)=I_{2 n}^{\prime}(0, \chi)
$$

If $0 \leq m_{0}<2 n+1$,

$$
R_{2 n}\left(V_{m_{0}}^{+}\right) \oplus R_{2 n}\left(V_{m_{0}}^{-}\right)=\operatorname{Im}\left(M_{2 n}^{*}\left(s_{0}, \chi\right)\right)=\operatorname{Ker}\left(M_{2 n}^{*}\left(-s_{0}, \chi\right)\right),
$$

is the maximal proper submodule of $I_{2 n}^{\prime}\left(-s_{0}, \chi\right)$. Here $M_{2 n}^{*}(s, \chi)$ is the normalized intertwining operator [9], [21]:

$$
M_{2 n}^{*}(s, \chi): I_{2 n}^{\prime}(s, \chi) \longrightarrow I_{2 n}^{\prime}(-s, \chi) .
$$

Moreover, the irreducible module

$$
X\left(s_{0}\right)=\operatorname{Im}\left(M_{2 n}^{*}\left(-s_{0}, \chi\right)\right) \simeq I_{2 n}^{\prime}\left(-s_{0}, \chi\right) /\left(R_{2 n}\left(V_{m_{0}}^{+}\right) \oplus R_{2 n}\left(V_{m_{0}}^{-}\right)\right)
$$

is the maximal proper submodule of $I_{2 n}^{\prime}\left(s_{0}, \chi\right)$, and

$$
X\left(s_{0}\right)=\operatorname{Ker}\left(M_{2 n}^{*}\left(s_{0}, \chi\right)\right) .
$$

Also, in the range $2 n+1<m \leq 4 n+2$, where $s_{0}=\frac{m}{2}-\rho_{2 n}$,

$$
\begin{gathered}
I_{2 n}^{\prime}\left(s_{0}, \chi\right)=R_{2 n}\left(V_{m}^{+}\right)+R_{2 n}\left(V_{m}^{-}\right), \\
X\left(s_{0}\right)=R_{2 n}\left(V_{m}^{+}\right) \cap R_{2 n}\left(V_{m}^{-}\right),
\end{gathered}
$$

and

$$
R_{2 n}\left(V_{m}^{ \pm}\right) / X\left(s_{0}\right) \simeq R_{2 n}\left(V_{m_{0}}^{ \pm}\right) .
$$

In the cases in which $V_{m_{0}}^{-}$does not exist, this last relation means that $X\left(s_{0}\right)=$ $R_{2 n}\left(V_{m}^{-}\right)$.

Now, turning to the proof of Theorem 3.9, we note that $m_{\chi}^{+}(\pi)=0$ implies that $\pi=\mathbb{1}$, so this case is covered by Lemma 4.2. Also note that, (4.10), together with Proposition 4.1 and our hypothesis on $\pi$, imply that

$$
\min \left\{m_{\chi}^{+}(\pi), m_{\chi}^{-}(\pi)\right\} \leq \begin{cases}2 n+1 & \text { for odd parity } \\ 2 n+2 & \text { for even parity. }\end{cases}
$$


Similarly, the fact that $I_{2 n}^{\prime}\left(s_{0}, \chi\right)$ is irreducible in the cases (i) $s_{0}=n+1$ and arbitrary $\chi$ for odd parity, (ii) $s_{0}=n+\frac{1}{2}$ and $\chi \neq 1$ and (iii) $s_{0}=n+\frac{3}{2}$ and $\chi=1$, for even parity, implies that

$$
\max \left\{m_{\chi}^{+}(\pi), m_{\chi}^{-}(\pi)\right\} \leq \begin{cases}4 n+3 & \text { for odd parity, } \\ 4 n+2 & \text { for even parity with } \chi \neq 1, \text { and } \\ 4 n+4 & \text { for even parity with } \chi=1\end{cases}
$$

These bounds are a little weaker than the stable range condition of Lemma 3.3 (ii).

We suppose that $\epsilon= \pm 1$ is taken so that $m_{\chi}^{\epsilon}(\pi)=\min \left\{m_{\chi}^{+}(\pi), m_{\chi}^{-}(\pi)\right\}$.

In the cases $m_{\chi}^{\epsilon}(\pi)=m_{0}$ with $m_{0}=1$ and $\chi$ arbitrary, (resp. $m_{0}=2$ with $\chi \neq 1$ ), we must have $m_{\chi}^{-\epsilon}(\pi) \geq 4 n+3$ (resp. $m_{\chi}^{-\epsilon}(\pi) \geq 4 n+2$ ) by Theorem 3.8. The upper bounds just described then force $m_{\chi}^{-\epsilon}(\pi)=4 n+3$ (resp. $m_{\chi}^{-\epsilon}(\pi)=4 n+2$ ), as claimed.

Next, suppose that $2 \leq m_{\chi}^{\epsilon}(\pi)=m_{0} \leq 2 n+1$. By Theorem 3.8, this forces $m_{\chi}^{-\epsilon}(\pi) \geq 4 n+4-m_{0} \geq 2 n+3$. With the conventions about complementary spaces just introduced, the minimality of $m_{\chi}^{\epsilon}(\pi)$ implies that

$$
\operatorname{dim} \operatorname{Hom}_{G^{\prime} \times G^{\prime}}\left(R_{2 n}\left(V_{m_{0}-2}^{+}\right) \oplus R_{2 n}\left(V_{m_{0}-2}^{-}\right), \pi \otimes \pi^{\vee}\right)=0 .
$$

Thus, every element of $\operatorname{Hom}_{G^{\prime} \times G^{\prime}}\left(I_{2 n}^{\prime}\left(-s_{0}-1, \chi\right), \pi \otimes \pi^{\vee}\right)$ must factor through $X\left(s_{0}+1\right)$, and

$$
\operatorname{dim} \operatorname{Hom}_{G^{\prime} \times G^{\prime}}\left(X\left(s_{0}+1\right), \pi \otimes \pi^{\vee}\right)=1 .
$$

On the other hand, let

$$
\mu \in \operatorname{Hom}_{G^{\prime} \times G^{\prime}}\left(I_{2 n}^{\prime}\left(s_{0}+1, \chi\right), \pi \otimes \pi^{\vee}\right)
$$

be a nonzero element. The restriction of $\mu$ to $X\left(s_{0}+1\right)$ must be nonzero, since, otherwise, it would factor through the quotient $R_{2 n}\left(V_{m_{0}-2}^{+}\right) \oplus R_{2 n}\left(V_{m_{0}-2}^{-}\right)$, contradicting (4.13). But, in the range in question, $X\left(s_{0}+1\right) \subset R_{2 n}\left(V_{m+2}^{-\epsilon}\right)$ is a nonzero submodule, and hence

$$
\left.\mu\right|_{R_{2 n}\left(V_{m+2}^{-\epsilon}\right)} \neq 0 .
$$

Thus

$$
m_{\chi}^{-\epsilon}(\pi) \leq m+2=4 n+4-m_{0} .
$$

This yields the equality $m_{\chi}^{+}(\pi)+m_{\chi}^{-}(\pi)=4 n+4$ in this case.

Only the case $m_{\chi}^{\epsilon}(\pi)=2 n+2$ remains. Let

$$
\mu \in \operatorname{Hom}_{G^{\prime} \times G^{\prime}}\left(I_{2 n}^{\prime}\left(\frac{1}{2}, \chi\right), \pi \otimes \pi^{\vee}\right)
$$


be a nonzero element, and note that the restriction of $\mu$ to the submodule $X\left(\frac{1}{2}\right)$ cannot be zero, since $\mu$ does not factor through the quotient

$$
I_{2 n}\left(\frac{1}{2}, \chi\right) / X\left(\frac{1}{2}\right) \simeq R_{2 n}\left(V_{2 n}^{+}\right) \oplus R_{2 n}\left(V_{2 n}^{-}\right) .
$$

But then, the restriction of $\mu$ to $R_{2 n}\left(V_{2 n+2}^{-\epsilon}\right) \supset X\left(\frac{1}{2}\right)$ is nonzero, as required.

Remark 4.4. An examination of our proof confirms that we have not made use of the stable range result Lemma 3.3 (ii), and that we have in fact reproved this fact.

\section{Proof of (ii) of Proposition 1.6: simplicity of poles}

In this section, we give the proof of the following result, which is just a restatement of part (ii) of Proposition 1.6. Note that we may absorb the unramified character $\chi$ into $s$ by a suitable shift, so that it can be omitted in what follows. Also, for convenience, we will write $\Phi_{s}$ rather than $\Phi(s)$ for a given standard section.

Proposition 5.1. Let $\mathfrak{z}_{s} \in \mathcal{H}(G / / K) \otimes \mathbb{C}\left[q^{s}, q^{-s}\right]$ be the element defined by

$$
\mathfrak{z}_{s}=\prod_{i=1}^{n}\left(1-q^{-s-\frac{1}{2}} t_{i}\right)\left(1-q^{-s-\frac{1}{2}} t_{i}^{-1}\right) .
$$

For an unramified representation $\pi$ of $G$, let $\pi\left(\mathfrak{z}_{s}\right)$ be the scalar by which $\mathfrak{z}_{s}$ acts on the unramified vector in $\pi$. Then for all matrix coefficients $\phi$ of $\pi^{\vee}$ and all standard sections $\Phi(s) \in I_{2 n}(s)$, the function

$$
\pi\left(\mathfrak{z}_{s}\right) \cdot Z\left(s, \chi_{0}, \phi, \Phi\right)
$$

is an entire function of $s$.

Proof. The proof will be broken into several steps.

Step 1. By linearity, it is sufficient to consider matrix coefficients of the form

$$
\phi(g)=<\pi^{\vee}(g) \pi^{\vee}\left(g_{0}\right) \xi_{0}^{\vee}, \pi\left(g_{1}\right) \xi_{0}>,
$$


where $\xi_{0}^{\vee}$ and $\xi_{0}$ are spherical vectors in $\pi^{\vee}$ and $\pi$, as above and $g_{0}$ and $g_{1} \in G$. Then

$$
\begin{aligned}
Z(s, \phi, \Phi) & =\int_{G} \Phi_{s}\left(\delta_{0} \iota(g, 1)\right) \cdot<\pi^{\vee}(g) \pi^{\vee}\left(g_{0}\right) \xi_{0}^{\vee}, \pi\left(g_{1}\right) \xi_{0}>d g \\
& =\int_{G} \Phi_{s}\left(\delta_{0} \iota\left(g_{1} g g_{0}^{-1}, 1\right)\right) \cdot<\pi^{\vee}(g) \xi_{0}^{\vee}, \xi_{0}>d g \\
& =\int_{G} \Phi_{s}\left(\delta_{0} \iota(g, 1) \iota\left(g_{0}^{-1}, g_{1}^{-1}\right)\right) \cdot \phi^{0}(g) d g .
\end{aligned}
$$

Since $\phi^{0}$ is bi- $K$-invariant, the last integral here is equal to

$$
\begin{aligned}
& \int_{G} \Phi_{s}\left(\delta_{0} \iota\left(k_{1}^{-1} g k_{2}, 1\right) \iota\left(g_{0}^{-1}, g_{1}^{-1}\right)\right) \cdot \phi^{0}(g) d g \\
= & \int_{G} \Phi_{s}\left(\delta_{0} \iota(g, 1) \iota\left(k_{1}, k_{2}\right) \iota\left(g_{0}^{-1}, g_{1}^{-1}\right)\right) \cdot \phi^{0}(g) d g .
\end{aligned}
$$

Thus we have

$$
Z(s, \phi, \Phi)=\int_{G} \Psi_{s}\left(\delta_{0} \iota(g, 1)\right) \cdot \phi^{0}(g) d g,
$$

where, for any $h \in H=G_{2 n}$,

$$
\Psi_{s}(h):=\int_{K \times K} \Phi_{s}\left(h \iota\left(k_{1}, k_{2}\right) \iota\left(g_{0}^{-1}, g_{1}^{-1}\right)\right) d k_{1} d k_{2} .
$$

Note that $\Psi_{s}$ is again a section of $I_{2 n}(s)$ which is right $K \times K$-invariant. It may not be standard, however!

Step 2. We next define an element $\mathfrak{z}$ of the algebra

$$
\mathcal{A}=\mathbb{C}\left[X, X^{-1}\right] \otimes \mathcal{H}(G / / K) \simeq \mathbb{C}\left[X, X^{-1}\right] \otimes \mathbb{C}\left[t_{1}, t_{1}^{-1}, \ldots, t_{n}, t_{n}^{-1}\right]^{W_{G}},
$$
where $\mathcal{H}(G / / K)$ is the $K$-spherical Hecke algebra of $G$, by

$$
\mathfrak{z}=\prod_{i=1}^{n}\left(1-q^{-\frac{1}{2}} X t_{i}\right)\left(1-q^{-\frac{1}{2}} X t_{i}^{-1}\right) .
$$

The algebra $\mathcal{A}$ acts on the space $I_{2 n}(s)^{K \times 1}$ of $K \times 1$-invariant vectors in $I_{2 n}(s)$ where the action of $\mathcal{H}(G / / K)$ comes from the action of $G \times 1$, via $\iota$, and where $X$ acts by scalar multiplication by $q^{-s}$. Note that this action commutes with the action of $1 \times G$. The operator $\mathfrak{z}$ is defined to kill support of sections.

Proposition 5.2. For any standard section $\Phi_{s}$ with associated section $\Psi_{s}$ defined by (5.5)

$$
\Psi_{s} * \mathfrak{z} \in I_{2 n}^{(0)}(s)^{K \times K}
$$


Proof. To illustrate the argument, we first consider the restriction to the closed orbit,

$$
\operatorname{pr}_{n}: I_{2 n}(s)=I_{2 n}^{(n)}(s) \longrightarrow Q_{n}(s) \simeq I_{n}\left(s+\frac{n}{2}\right) \otimes I_{n}\left(s+\frac{n}{2}\right),
$$

given by $\Phi_{s} \mapsto\left(\left(g_{1}, g_{2}\right) \mapsto \Phi_{s}\left(\iota\left(g_{1}, g_{2}\right)\right)\right)$, the map obtained by restriction of sections to $\Omega_{n}$. Then we have

$$
\operatorname{pr}_{n}\left(\Psi_{s} * \mathfrak{z}\right)=\operatorname{pr}_{n}\left(\Psi_{s}\right) * \mathfrak{z},
$$

where $\mathfrak{z}$ is acting on the $K$-invariant vectors in the first factor of the tensor product on the right side of (5.8). Thus, it suffices to prove that $\mathfrak{z}$ acts by zero on the space $I_{n}\left(s+\frac{n}{2}\right)^{K}$. But

$$
I_{n}\left(s+\frac{n}{2}\right) \subset I_{B}^{G}(\lambda)
$$

where $B$ is the standard Borel subgroup of $G$ and $I_{B}^{G}(\lambda)$ is the unramified principal series representation of $G$ with Satake parameter

$$
\lambda=\left(q^{-s-\frac{1}{2}}, q^{-s-\frac{3}{2}}, \ldots, q^{-s-n+\frac{1}{2}}\right) .
$$

The operator $\mathfrak{z}$ then acts on the (common) $K$-fixed vector in these representations by the scalar

$$
\prod_{i=1}^{n}\left(1-q^{-s-\frac{1}{2}} q^{-s-i+\frac{1}{2}}\right)\left(1-q^{-s-\frac{1}{2}} q^{s+i-\frac{1}{2}}\right)=0 .
$$

Thus $\operatorname{pr}_{n}\left(\Psi_{s} * \mathfrak{z}\right)=0$ so that $\Psi_{s} * \mathfrak{z} \in I_{2 n}^{(n-1)}(s)$.

In general, restriction of sections to the orbit $\Omega_{r}$ yields a map

$$
\operatorname{pr}_{r}: I_{2 n}(s) \longrightarrow I_{P_{r} \times P_{r}}^{G \times G}\left(||^{s+\frac{r}{2}} \otimes||^{s+\frac{r}{2}} \otimes C\left(G_{n-r}\right)\right)=: B_{r}(s),
$$

where $C\left(G_{n-r}\right)$ is the space of all smooth functions on $G_{n-r}$. There is a nondegenerate pairing

$$
Q_{r}(s) \otimes B_{r}(-s-r) \longrightarrow \mathbb{C}
$$

given by

$$
\left\langle f_{1}, f_{2}\right\rangle=\int_{P_{r} \times P_{r} \backslash G \times G}\left\langle f_{1}\left(g_{1}, g_{2}\right), f_{2}\left(g_{1}, g_{2}\right)\right\rangle_{G_{n-r}} d \mu\left(g_{1}\right) d \mu\left(g_{2}\right)
$$

where the integrand is the pairing on the values of function $f_{1} \in B_{r}(-s-r)$ and $f_{2} \in Q_{r}(s)$ given by integration over $G_{n-r}$ and the outer 'integral' is the $G \times G$ invariant functional on the spaces of functions on $G \times G$ transforming on the left under $P_{r} \times P_{r}$ according to the square of the modulus character. A straightforward density argument shows that an element $\phi \in Q_{r}(s)$ is zero if and only if it pairs to zero against all elements of the subspace $Q_{r}(-s-r)$ 
of $B_{r}(-s-r)$. If $\phi \in Q_{r}(s)^{K \times K}$, then it suffices to pair with elements $f \in$ $Q_{r}(-s-r)^{K \times K}$.

We consider the pairing

$$
\left\langle\operatorname{pr}\left(\Psi_{s} * \mathfrak{z}\right), f_{s}\right\rangle=\left\langle\operatorname{pr}\left(\Psi_{s}\right), f_{s} * \mathfrak{z}_{s}^{\vee}\right\rangle
$$

for any function $f_{s} \in Q_{r}(-s-r)^{K \times K}$, where $\mathfrak{z}_{s}^{\vee}$ is the adjoint of $\mathfrak{z}_{s}=\left.\mathfrak{z}\right|_{X=q^{-s}}$.

Lemma 5.3. For any function $f_{s} \in Q_{r}(-s-r)^{K \times K}$,

$$
f_{s} * \mathfrak{z}_{s}^{\vee}=0 \text {. }
$$

Proof. Since a function in $f_{s} \in Q_{r}(-s-r)^{K \times K}$ is determined by its value at the identity element, we can consider $f_{s}(e) \in S\left(G_{n-r}\right)$. Note that, for $x \in G_{n-r}$ and $k_{1}, k_{2} \in K_{n-r}=K \cap G_{n-r}$, we have

$$
f_{s}(e)\left(k_{1}^{-1} x k_{2}\right)=f_{s}\left(k_{1}, k_{2}\right)(x)=f_{s}(e)(x),
$$

so that, in fact,

$$
f_{s}(e) \in S\left(G_{n-r}\right)^{K_{n-r} \times K_{n-r}} .
$$

If $\tau$ is any irreducible admissible representation of $G_{n-r}$, the action of the space of functions $S\left(G_{n-r}\right)$ on $\tau$ determines a $G_{n-r} \times G_{n-r}$-equivariant map

$$
\mu_{\tau}: S\left(G_{n-r}\right) \longrightarrow \operatorname{Hom}^{\operatorname{smooth}}(\tau, \tau) \simeq \tau^{\vee} \otimes \tau .
$$

Here, note that $G \times G$ acts on $\operatorname{Hom}(\tau, \tau)$ by pre and pos-multiplication, so that elements of $\operatorname{Hom}^{\text {smooth }}(\tau, \tau)$, which by definition are fixed by some compact open subgroup $K \times K$, have finite dimensional image. A function $\phi \in S\left(G_{n-r}\right)^{K_{n-r} \times K_{n-r}}$ is nonzero if and only if there is an unramified representation $\tau$ of $G_{n-r}$ such that $\tau(\phi) \neq 0$, i.e., $\mu_{\tau}(\phi) \neq 0$.

Applying this to $f_{s} * \mathfrak{z}_{s}^{\vee}$, we suppose that there is an irreducible unramified representation $\tau$ of $G_{n-r}$ such that

$$
\mu_{\tau}\left(f_{s} * \mathfrak{z}_{s}^{\vee}(e)\right) \neq 0 .
$$

There is a map of induced representations

$$
\begin{aligned}
I\left(\mu_{\tau}\right): I_{P_{r} \times P_{r}}^{G \times G}\left(||^{-s-\frac{r}{2}} \otimes||^{-s-\frac{r}{2}} \otimes S\left(G_{n-r}\right)\right) & \\
& \longrightarrow I_{P_{r} \times P_{r}}^{G \times G}\left(||^{-s-\frac{r}{2}} \otimes||^{-s-\frac{r}{2}} \otimes \tau^{\vee} \otimes \tau\right),
\end{aligned}
$$

and

$$
I\left(\mu_{\tau}\right)(f)(e)=\mu_{\tau}(f(e)) .
$$


We can embed the latter of these representations into a full induced representation

$$
I_{P_{r}}^{G}\left(||^{-s-\frac{r}{2}} \otimes \tau^{\vee}\right) \otimes I_{P_{r}}^{G}\left(||^{-s-\frac{r}{2}} \otimes \tau\right) \quad \hookrightarrow \quad I_{B}^{G}\left(\lambda_{1}\right) \otimes I_{B}^{G}\left(\lambda_{2}\right),
$$

where the Satake parameter $\lambda_{1}$ is given by

$$
\lambda_{1}=\left(q^{s+r-\frac{1}{2}}, q^{s+r-\frac{3}{2}}, \ldots, q^{s+\frac{1}{2}}, q^{\nu_{1}}, \ldots, q^{\nu_{n-r}}\right) .
$$

Note that $\mathfrak{z}_{s}^{\vee}=\mathfrak{z}_{s}$ acts on the unique $K$-invariant vector in this representation by the scalar

$$
\prod_{i=1}^{r}\left(1-q^{-s-\frac{1}{2}} q^{s+r+\frac{1}{2}-i}\right)\left(1-q^{-s-\frac{1}{2}} q^{-s-r-\frac{1}{2}+i}\right) \cdot(\star)=0 .
$$

But then, we have

$$
\begin{aligned}
\mu_{\tau}\left(f_{s} * \mathfrak{z}_{s}^{\vee}(e)\right) & =I\left(\mu_{\tau}\right)\left(f_{s} * \mathfrak{z}_{s}^{\vee}\right)(e) \\
& =\left(I\left(\mu_{\tau}\right)\left(f_{s}\right) * \mathfrak{z}_{s}^{\vee}\right)(e) \\
& =0,
\end{aligned}
$$

since $I\left(\mu_{\tau}\right)\left(f_{s}\right)$ is a multiple of the $K \times K$-invariant vector in $I_{B}^{G}\left(\lambda_{1}\right) \otimes I_{B}^{G}\left(\lambda_{2}\right)$. This finishes the proof of Lemma 5.3 and hence of Proposition 5.2.

Step 3. Consider the isomorphism

$$
\operatorname{pr}_{0}: I_{2 n}^{(0)}(s) \longrightarrow Q_{0}(s) \simeq S(G),
$$

given by $\Phi_{s} \mapsto\left((g, 1) \mapsto \Phi_{s}\left(\delta_{0} \iota(g, 1)\right)\right)$. Proposition 1.7 shows that, for a fixed $s$, the image of $\Psi_{s} * \mathfrak{z}$ under $\operatorname{pr}_{0}$ lies in $S(G)^{K \times K}$. But the support could, a priori, vary with $s$. The following result shows that the support is, in fact, bounded uniformly in $s$.

\section{Lemma 5.4.}

$$
\operatorname{pr}_{0}\left(\Psi_{s} * \mathfrak{z}\right) \in \mathbb{C}\left[q^{s}, q^{-s}\right] \otimes S(G)^{K \times K}=\mathbb{C}\left[q^{s}, q^{-s}\right] \otimes \mathcal{H}(G / / K) .
$$

Proof. Using the Cartan decomposition, write

$$
\operatorname{pr}_{0}\left(\Psi_{s} * \mathfrak{z}\right)=\sum_{\lambda \in \Lambda} c_{\lambda}(s) L_{\lambda},
$$

where $L_{\lambda}$ is the characteristic function of the double coset $K g_{\lambda} K$ and $\Lambda$ is the usual semigroup.

Lemma 5.5. For each $\lambda \in \Lambda$, the function $c_{\lambda}(s)$ is an entire analytic function of $s$. In fact, $c_{\lambda}(s) \in \mathbb{C}\left[q^{s}, q^{-s}\right]$. 
Assuming this result for the moment, let $\Lambda_{1}$ be the set of $\lambda$ 's for which the function $c_{\lambda} \neq 0$, and let

$$
D_{\lambda}=\left\{s \in \mathbb{C} \mid c_{\lambda}(s) \neq 0\right\} .
$$

For $\lambda \in \Lambda_{1}, D_{\lambda}$ is an open dense subset of $\mathbb{C}$. Thus, by the Baire category theorem, the set

$$
\bigcap_{\lambda \in \Lambda_{1}} D_{\lambda}
$$

is non-empty, and so, there is an $s_{0} \in \mathbb{C}$ such that $c_{\lambda}\left(s_{0}\right) \neq 0$ for all $\lambda \in \Lambda_{1}$. Since

$$
\operatorname{pr}_{0}\left(\Psi_{s_{0}} * \mathfrak{z}\right)=\sum_{\lambda \in \Lambda_{1}} c_{\lambda}\left(s_{0}\right) L_{\lambda}
$$

has compact support, it follows that the set $\Lambda_{1}$ is finite, as claimed!

Proof of Lemma 5.5. We have the formula

$$
c_{\lambda}(s) \cdot\left\|L_{\lambda}\right\|^{2}=\int_{G}\left(\Psi_{s} * \mathfrak{z}\right)\left(\delta_{0} \iota(g, 1)\right) \cdot L_{\lambda}(g) d g .
$$

The right side of (5.32) is a finite linear combination, with coefficients in $\mathbb{C}\left[q^{s}, q^{-s}\right]$, of integrals of the form

$$
\begin{aligned}
& \int_{G} \int_{G} \Psi_{s}\left(\delta_{0} \iota(g, 1) \iota\left(g_{0}, 1\right)\right) \cdot L_{\mu}\left(g_{0}\right) d g_{0} \cdot L_{\lambda}(g) d g \\
= & \int_{G} \int_{G} \Psi_{s}\left(\delta_{0} \iota\left(g_{0}, 1\right)\right) \cdot L_{\mu}\left(g^{-1} g_{0}\right) \cdot L_{\lambda}(g) d g_{0} d g \\
= & \int_{G} \Psi_{s}\left(\delta_{0} \iota\left(g_{0}, 1\right)\right) \cdot \varphi\left(g_{0}\right) d g_{0}
\end{aligned}
$$

where $\varphi \in \mathcal{H}(G / / K)$ is an element depending on $\lambda$ and $\mu$. Since $\varphi$ is a finite linear combination of characteristic functions of cosets $g K$, the integral in the last line of (5.33) is a finite linear combination of terms of the form

$$
\int_{K} \int_{K \times K} \Phi_{s}\left(\delta_{0} \iota(g k, 1) \iota\left(k_{1}, k_{2}\right) \iota\left(g_{1}, g_{2}\right)\right) d k_{1} d k_{2} d k .
$$

But, noting that $\left(g_{1}, g_{2}\right)$ is fixed and recalling that $\Phi_{s}$ is standard and hence is right invariant under some compact open subgroup of $H$, uniformly in $s$, this expression is just a finite linear combination of values of $\Phi_{s}$ at a set of points not depending on $s$. Such values have the form $c q^{r s}$ for $r \in \mathbb{Z}$. This finishes the proof of Lemma 5.5. 
Step 4. Now returning to the zeta integral (5.4) and inserting the operator $\mathfrak{z}$, we find that

$$
Z^{*}\left(s, \phi^{0}, \Psi\right):=\int_{G}\left(\Psi_{s} * \mathfrak{z}\right)\left(\delta_{0} \iota(g, 1)\right) \phi^{0}(g) d g
$$

is equal to the scalar by which the element $\operatorname{pr}_{0}\left(\Psi_{s} * \mathfrak{z}\right)$ of $\mathcal{A}$ acts on the spherical vector $\xi_{0}^{\vee}$ in $\pi^{\vee}$. This scalar lies in $\mathbb{C}\left[q^{s}, q^{-s}\right]$, and, in particular, is an entire function of $s$. On the other hand, for $\operatorname{Re}(s)$ large,

$$
\begin{aligned}
Z^{*}\left(s, \phi^{0}, \Psi\right) & =\pi^{\vee}\left(\mathfrak{z}_{s}\right) \cdot \int_{G} \Psi_{s}\left(\delta_{0} \iota(g, 1)\right) \phi^{0}(g) d g \\
& =\pi^{\vee}\left(\mathfrak{z}_{s}\right) \cdot Z(s, \phi, \Phi)
\end{aligned}
$$

where $\pi^{\vee}\left(\mathfrak{z}_{s}\right)$ is the scalar by which $\mathfrak{z}_{s}:=\left.\mathfrak{z}\right|_{X=q^{-s}}$ acts on the spherical vector in $\pi^{\vee}$. Since $Z^{*}\left(s, \phi^{0}, \Psi\right)$ is an entire function of $s$, this completes the proof of the proposition.

Note that

$$
\begin{aligned}
\pi^{\vee}\left(\mathfrak{z}_{s}\right) & =\prod_{i=0}^{n}\left(1-q^{-s-\frac{1}{2}} \pi^{\vee}\left(t_{i}\right)\right)\left(1-q^{-s-\frac{1}{2}} \pi^{\vee}\left(t_{i}^{-1}\right)\right) \\
& =b_{2 n}(s)^{-1}\left(1-q^{-s-\frac{1}{2}}\right)^{-1} \cdot Z\left(s, \phi^{0}, \Phi^{0}\right)^{-1} .
\end{aligned}
$$

Thus

$$
Z\left(s, \phi^{0}, \Phi^{0}\right)^{-1} \cdot Z(s, \phi, \Phi)=b_{2 n}(s)\left(1-q^{-s-\frac{1}{2}}\right) Z^{*}\left(s, \phi^{0}, \Psi_{s}\right) .
$$

This shows that the unramified zeta integral $Z\left(s, \phi^{0}, \Phi^{0}\right)$ can have some extra zeros, compared to the whole family of zeta integrals.

\section{References}

[1] F. Bruhat, Distributions sur un groupe localement compact et applications à l'étude des representations des groupes p-adiques, Bull. Soc. Math. France, 89 (1961), 43-75.

[2] R. Gustafson, The degenerate principal series of $S p(2 n)$, Memoirs of AMS. 248 (1981).

[3] M. Harris, S. Kudla, and W. Sweet, Theta dichotomy for unitary groups, Jour. of the AMS, 9 (1996), pp. 941-1004.

[4] R. Howe, $\theta$-series and invariant theory, in Automorphic Forms, Representations and $L$-functions, Proc. Symp. Pure Math., 33 (1979), pp. 275-285. 
[5] S. Kudla, On the local theta correspondence, Invent. math., 83 (1986), pp. 229-255.

[6] _ Splitting metaplectic covers of dual reductive pairs, Israel J. Math., 87 (1994), pp. 361-401.

[7] Notes on the local theta correspondence, Lecture notes from the European School on Group Theory, Schloß Hirschberg, Germany, 1996

[8] Central derivatives of Eisenstein series and height pairings, Ann. of Math., 146 (1997), pp. 545-646.

[9] S. Kudla and S. Rallis, Ramified degenerate principal series, Israel Math. J., 78 (1992), pp. 209-256.

[10] S. Kudla and W. Sweet, Degenerate principal series for unitary groups, Israel J. Math. 98 (1997), 253-306.

[11] E. Lapid and S. Rallis, On the local factors of classical groups, this volume.

[12] C. Moeglin and M. Tadic, Construction of discrete series for classical p-adic groups, Jour. of the AMS, 15 (2002), 715-786.

[13] C. Moeglin, M.-F. Vigneras, and J.-L. Waldspurger, Correspondances de Howe sur un corps p-adique, Lecture Notes in Math., 1291, Springer-Verlag, New York, 1987.

[14] G. Muiç, Howe correspondence for discrete series representations; the case of $(\mathrm{Sp}(\mathrm{n}), \mathrm{O}(\mathrm{V}))$, to appear in Crelle Math. J.

[15] A. Paul, First occurrence for the dual pairs $(U(p, q), U(r, s))$, Canadian J. Math. 51 (1999), 636-657.

[16] I. I. Piatetski-Shapiro and S. Rallis, $\epsilon$-factors of representations of classical groups, Proc. Nat. Acad. Sci., 83 (1986), pp. 4589-4593.

[17] I. I. Piatetski-Shapiro and S. Rallis, L-functions for classical groups, Lecture Notes in Math., 1254, Springer-Verlag, New York, 1987, pp. 1-52.

[18] S. Rallis, On the Howe duality conjecture, Compositio Math., 51 (1984), pp. 333-399.

[19] R. Rao, On some explicit formulas in the theory of the Weil representation, Pacific J. Math., 157 (1993), pp. 335-371.

[20] W. Sweet, A computation of the gamma matrix of a family of p-adic zeta integrals, J. of Number Theory, 55 (1995), 222-260.

[21] Functional equations of p-adic zeta integrals and representations of the metaplectic group, preprint, 1995.

[22] J.-L. Waldspurger, Demonstration d'une conjecture de duality de Howe dans le case $p$-adiques, $p \neq 2$, Festschrift in Honor of Piatetski-Shapiro, Israel Math. Conf. Proc., 2, 1990, pp. 267-324. 